\title{
Algebraic cycles and the classical groups Part II: Quaternionic cycles
}

\author{
H Blaine Lawson JR \\ Paulo Lima-Filho \\ MARie-Louise MiChelsohn \\ BL, MM: Department of Mathematics, Stony Brook University \\ Stony Brook, NY 11794, USA \\ PL: Department of Mathematics, Texas A\&M University \\ College Station, TX 77843, USA \\ blaine@math.sunysb.edu, plfilho@math.tamu.edu, mlm@math.sunysb.edu
}

\begin{abstract}
In part I of this work we studied the spaces of real algebraic cycles on a complex projective space $\mathbb{P}(V)$, where $V$ carries a real structure, and completely determined their homotopy type. We also extended some functors in $K$-theory to algebraic cycles, establishing a direct relationship to characteristic classes for the classical groups, specially Stiefel-Whitney classes. In this sequel, we establish corresponding results in the case where $V$ has a quaternionic structure. The determination of the homotopy type of quaternionic algebraic cycles is more involved than in the real case, but has a similarly simple description. The stabilized space of quaternionic algebraic cycles admits a nontrivial infinite loop space structure yielding, in particular, a delooping of the total Pontrjagin class map. This stabilized space is directly related to an extended notion of quaternionic spaces and bundles ( $K H$-theory), in analogy with Atiyah's real spaces and $K R$-theory, and the characteristic classes that we introduce for these objects are nontrivial. The paper ends with various examples and applications.
\end{abstract}

\section{AMS Classification numbers Primary: 14C25}

Secondary: 55P43, 14P99, 19L99, 55P47, 55P91

Keywords: Quaternionic algebraic cycles, characteristic classes, equivariant infinite loop spaces, quaternionic $K$-theory

Proposed: Ralph Cohen

Received: 24 April 2002

Seconded: Gunnar Carlsson, Haynes Miller

Revised: 28 April 2005 


\section{Introduction}

In Part 1 [15] of this paper we studied the spaces of real algebraic cycles in $\mathbb{P}\left(\mathbb{C}^{n}\right)$ and found that their homotopy structure was particularly simple and surprisingly related to the Stiefel-Whitney and Pontrjagin classes. We saw that the stabilized space $\mathcal{Z}_{\mathbb{R}}^{\infty}$ of all such cycles is an $E_{\infty}$-ring space whose homotopy groups $\pi_{*} \mathcal{Z}_{\mathbb{R}}^{\infty}$ form a graded ring isomorphic to $\mathbb{Z}[x, y] /(2 y)$. Furthermore, the standard complexification and forgetful functors in $K$-theory were shown to extend over the characteristic homomorphisms to infinite loop maps of cycle spaces. Here in Part 2 we shall establish analogous results for spaces of quaternionic cycles.

Recall that a real vector space is a pair $(V, \rho)$ where $V$ is a complex vector space and $\rho: V \rightarrow V$ is an anti-linear map with $\rho^{2}=\mathrm{Id}$. A real algebraic subvariety (or more generally a real algebraic cycle) in $\mathbb{P}(V)$ is one which is fixed by the induced involution $\rho: \mathbb{P}(V) \rightarrow \mathbb{P}(V)$ (the action of $\operatorname{Gal}(\mathbb{C} / \mathbb{R})$ ). This condition is equivalent to assuming that the subvariety is defined by real algebraic equations.

Analogously a quaternionic vector space is a pair $(V, \mathbf{j})$ where $\mathbf{j}: V \rightarrow V$ is an anti-linear map with $\mathbf{j}^{2}=-\mathrm{Id}$. A quaternionic algebraic variety (or cycle) in $\mathbb{P}(V)$ is one which is fixed by the induced involution. Such subvarieties are distinctly different from real ones. The map $\mathbf{j}$ is fixed-point free on $\mathbb{P}(V)$ and therefore on every subvariety. The induced antilinear bundle map on $\mathcal{O}(1)$ has square - Id.

In 14 we prove that the quaternionic suspension of cycles gives a $\mathbb{Z}_{2}$-homotopy equivalence $\mathbb{Z}_{\mathbb{H}}: \mathcal{Z}^{q}(\mathbb{P}(V)) \rightarrow \mathcal{Z}^{q}(\mathbb{P}(V \oplus \mathbb{H}))$ of cycle spaces and in particular a homotopy equivalence

$$
\mathbb{Z}_{\mathbb{H}}: \mathcal{Z}_{\mathbb{H}}^{q}(\mathbb{P}(V)) \longrightarrow \mathcal{Z}_{\mathbb{H}}^{q}(\mathbb{P}(V \oplus \mathbb{H}))
$$

of the subgroups of codimension- $q$ quaternionic cycles. For $q$ odd one can thereby reduce to 0-cycles and apply Dold-Thom [3, 19, to determine the structure of these spaces. However, for $q$ even one can only reduce to 1-cycles by this method. The determination of the homotopy type of $\mathcal{Z}_{\mathbb{H}}^{q}$ when $q$ is even, is one of the main results of this paper (Theorem 2.3). Its proof, which involves new constructions and techniques, is given in Section [6]

Under stabilization there are two limits, $\mathcal{Z}_{\mathbb{H}}^{2 \infty}$ and $\mathcal{Z}_{\mathbb{H}}^{2 \infty+1}$, whose homotopy groups together form a $\mathbb{Z}_{2} \times \mathbb{Z}$-graded ring under the algebraic join pairing. Our second result is the determination of this ring which turns out to be quite simple. (See Theorem 3.4) 
In analogy with the real and complex cases, we then show that the inclusion of linear quaternionic cycles (the quaternionic Grassmannian) into the space of all cycles yields the characteristic map BSp $\longrightarrow \prod_{i} K(\mathbb{Z}, 4 i)$ classifying the total Pontrjagin class. Furthermore, the forgetful functor and the quaternionification functors from $K$-theory are shown to extend over the characteristic maps to infinite loop maps of cycles spaces.

It turns out that the spaces $\mathcal{Z}_{\mathbb{H}}^{2 \infty}$ and $\mathcal{Z}_{\mathbb{H}}^{2 \infty+1}$ have a second, more mysterious $\mathbb{Z}_{2}$-component which is not seen by the characteristic map from BSp. However, there is an extended notion of quaternionic spaces and bundles, in analogy with Atiyah's notion of real spaces and real bundles [1; and for such creatures our new $\mathbb{Z}_{2}$-characteristic classes are nontrivial. This is discussed at the end of the paper where examples and applications are given.

\section{Spaces of quaternionic cycles}

A quaternionic structure on a complex vector space $V$ is a $\mathbb{C}$-antilinear map $\mathbf{j}: V \rightarrow V$ such that $\mathbf{j}^{2}=-1$. A quaternionic vector space is a pair $(V, \mathbf{j})$ consisting of a complex vector space $V$ and a quaternionic structure $\mathbf{j}$. Any quaternionic vector space is equivalent to $\left(\mathbb{H}^{n}, \mathbf{j}_{0}\right)$ where $\mathbf{j}_{0}$ is left scalar multiplication by the quaternion $j$.

A quaternionic structure $\mathbf{j}$ on $V$ induces a free anti-holomorphic involution $\mathbf{j}: \mathbb{P}(V) \rightarrow \mathbb{P}(V)$ which can be viewed as follows. Let $\pi: \mathbb{P}(V) \rightarrow \mathbb{P}_{\mathbb{H}}(V)$ be the projection from the complex to the quaternionic projective space of $V$ whose fibres are projective lines. Then $\mathbf{j}$ preserves the fibres of $\pi$ and acts on them as the antipodal map on $S^{2}$. This map $\mathbf{j}$ induces an anti-holomorphic involution on the Chow varieties $\mathcal{C}_{d}^{q}(\mathbb{P}(V))$, which in turn induces an automorphism

$$
\mathbf{j}: \mathcal{Z}^{q}(\mathbb{P}(V)) \rightarrow \mathcal{Z}^{q}(\mathbb{P}(V)) .
$$

of the topological group of all codimension- $q$ cycles on $\mathbb{P}(V)$.

Definition 2.1 Let $(V, \mathbf{j})$ be a quaternionic vector space. Then the group $\mathcal{Z}_{\mathbb{H}}^{q}(\mathbb{P}(V))$ of quaternionic algebraic cycles of codimension $q$ on $\mathbb{P}(V)$, is the fixed point set of the involution (11). It contains the closed subgroup of averaged cycles $\mathcal{Z}^{q}(\mathbb{P}(V))^{\text {av }}=\left\{c+\mathbf{j} c \mid c \in \mathcal{Z}^{q}(\mathbb{P}(V))\right\}$. We define the group of reduced quaternionic algebraic cycles to be the quotient

$$
\widetilde{\mathcal{Z}}_{\mathbb{H}}^{q}(\mathbb{P}(V))=\mathcal{Z}_{\mathbb{H}}^{q}(\mathbb{P}(V)) / \mathcal{Z}^{q}(\mathbb{P}(V))^{\text {av }} .
$$

Note that $\widetilde{\mathcal{Z}}_{\mathbb{H}}^{q}(\mathbb{P}(V))$ is the topological $\mathbb{Z}_{2}$-vector space freely generated by the $\mathbf{j}$-invariant irreducible subvarieties of codimension- $q$ in $\mathbb{P}(V)$. 
Example When $q=\operatorname{dim}(V)-2$, so that the cycle dimension is 1 , then the basis elements of $\widetilde{\mathcal{Z}}_{\mathbb{H}}^{q}(\mathbb{P}(V))$ are irreducible, $\mathbf{j}$-invariant algebraic curves. Note that if $C \subset \mathbb{P}(V)$ is such a curve, then the quotient $C_{0}=C / \mathbb{Z}_{2}$ of $C$ by $\mathbf{j}$ is a "nonorientable" algebraic curve carrying a "nonorientable" conformal structure. Conversely, any such creature $C_{0}$ has a $\mathbb{Z}_{2}$-covering $C \rightarrow C_{0}$ by a complex analytic curve where the covering involution $\mathbf{j}: C \rightarrow C$ is anti-holomorphic and free. The natural embedding spaces for such objects are $(\mathbb{P}(V), \mathbf{j})$ where $V$ is quaternionic. Thus one could think of $\widetilde{\mathcal{Z}}_{\mathbb{H}}^{q}\left(\mathbb{P}\left(\mathbb{H}^{2}\right)\right)$ as the topological $\mathbb{Z}_{2}$ vector space generated by the irreducible "nonorientable algebraic curves" in $\mathbb{P}^{3}$.

Given a quaternionic vector space $(V, \mathbf{j})$ and an algebraic subvariety $Z \subset \mathbb{P}(V)$, we define the quaternionic algebraic suspension $\mathbb{Z}_{\mathbb{H}}(Z) \subset \mathbb{P}(V \oplus \mathbb{H})$ to be the union of all complex projective lines joining $Z$ to $\mathbb{P}(\{0\} \oplus \mathbb{H})$. This determines a continuous $\mathbb{Z}_{2}$-equivariant homomorphism (cf [14, Section 6])

$$
\mathbb{Z}_{\mathbb{H}}: \mathcal{Z}^{q}(\mathbb{P}(V)) \longrightarrow \mathcal{Z}^{q}(\mathbb{P}(V \oplus \mathbb{H})) \text {. }
$$

Theorem 2.2 14, Theorem 6.1] The quaternionic algebraic suspension homomorphism (2) is a $\mathbb{Z}_{2}$-homotopy equivalence. It induces homotopy equivalences

and

$$
\begin{aligned}
& \mathbb{Z}_{\mathbb{H}}: \mathcal{Z}_{\mathbb{H}}^{q}(\mathbb{P}(V)) \stackrel{\cong}{\longrightarrow} \mathcal{Z}_{\mathbb{H}}^{q}(\mathbb{P}(V \oplus \mathbb{H})) \\
& \mathbb{Z}_{\mathbb{H}}: \widetilde{\mathcal{Z}}_{\mathbb{H}}^{q}(\mathbb{P}(V)) \stackrel{\cong}{\longrightarrow} \widetilde{\mathcal{Z}}_{\mathbb{H}}^{q}(\mathbb{P}(V \oplus \mathbb{H}))
\end{aligned}
$$

for all $q<\operatorname{dim}_{\mathbb{C}}(V)$.

Thus the homotopy types of the spaces $\mathcal{Z}_{\mathbb{H}}^{q}(\mathbb{P}(V))$ and $\widetilde{\mathcal{Z}}_{\mathbb{H}}^{q}(\mathbb{P}(V))$ depend only on $q$. One of our main results is the following computation of these homotopy types. The proof is given in Section 6 .

Theorem 2.3 For any quaternionic vector space $(V, \rho)$ there are canonical homotopy equivalences:

(i) $\mathcal{Z}_{\mathbb{H}}^{2 q}(\mathbb{P}(V)) \cong \prod_{j=0}^{q} K(\mathbb{Z}, 4 j) \times \prod_{j=1}^{q} K\left(\mathbb{Z}_{2}, 4 j-2\right)$ and $\widetilde{\mathcal{Z}}_{\mathbb{H}}^{2 q}(\mathbb{P}(V)) \cong \mathbb{Z}_{2}$,

(ii) $\mathcal{Z}_{\mathbb{H}}^{2 q+1}(\mathbb{P}(V)) \cong \prod_{j=0}^{q} K(\mathbb{Z}, 4 j) \times \prod_{j=0}^{q} K\left(\mathbb{Z}_{2}, 4 j+1\right)$ and $\widetilde{\mathcal{Z}}_{\mathbb{H}}^{2 q+1}(\mathbb{P}(V)) \cong$ $\{$ point $\}$ for all $q$.

Note that $\mathbb{Z}_{\mathbb{H}}$ changes cycle dimension by 2 . Therefore, for cycles of odd codimension we can de-suspend to the case of 0 -cycles and apply the Dold-Thom Theorem. For cycles of even codimension one must take a complex suspension by $\mathcal{O}(2)$ and then de-suspend the resulting spaces quaternionically. This latter argument is delicate and uses nontrivial results from the theory of cycles on quasi-projective varieties. This is all done in Section 6 . 


\section{$3 \quad$ Stabilization and the ring structure}

In this section we examine the limit over $V \subset \mathbb{H}^{\infty}$ of the cycles spaces $\mathcal{Z}_{\mathbb{H}}^{q}(V)$. There are two series: $q$ even and $q$ odd, with different limits. The algebraic join induces a product on the homotopy groups of these spaces, and we shall compute structure of the resulting $\mathbb{Z}_{2}$-ring. We begin with the following.

Proposition 3.1 Let $V$ and $W$ be quaternionic vector spaces of complex dimensions $2 v$ and $2 w$ respectively. Then for each $q$ with $0<q<2 v$, the inclusion

$$
\mathcal{Z}_{\mathbb{H}}^{q}(\mathbb{P}(V)) \subset \mathcal{Z}_{\mathbb{H}}^{q+2 w}(\mathbb{P}(V \oplus W))
$$

induces an injection on homotopy groups.

Proof There are two cases to consider: $q$ even and $q$ odd. The arguments are analogous. In both cases one reduces to 0 -cycles by quaternionic suspension: Corollary 6.4 in the even case and Theorem 2.2 in the odd case. Then by applying the Dold-Thom Theorem [3] to 0-cycles, it suffices to show that the maps in homology

$$
H_{*}\left(\mathbb{P}(V) / \mathbb{Z}_{2} ; \mathbb{Z}\right) \longrightarrow H_{*}\left(\mathbb{P}(V \oplus W) / \mathbb{Z}_{2} ; \mathbb{Z}\right)
$$

and

$$
H_{*}\left(Q(V) / \mathbb{Z}_{2} ; \mathbb{Z}\right) \longrightarrow H_{*}\left(Q(V \oplus W) / \mathbb{Z}_{2} ; \mathbb{Z}\right),
$$

where $Q(V)$ is defined in (20), are injective. This is straightforward.

Corollary 3.2 Consider the limiting spaces

$$
\mathcal{Z}_{\mathbb{H}}^{\mathrm{ev}}=\lim _{n, q \rightarrow \infty} \mathcal{Z}_{\mathbb{H}}^{2 q}\left(\mathbb{P}_{\mathbb{C}}\left(\mathbb{H}^{n}\right)\right) \quad \text { and } \quad \mathcal{Z}_{\mathbb{H}}^{\text {odd }}=\lim _{n, q \rightarrow \infty} \mathcal{Z}_{\mathbb{H}}^{2 q+1}\left(\mathbb{P}_{\mathbb{C}}\left(\mathbb{H}^{n}\right)\right)
$$

There are canonical homotopy equivalences:

$$
\begin{aligned}
\mathcal{Z}_{\mathbb{H}}^{\mathrm{ev}} & \cong \prod_{j=0}^{\infty} K(\mathbb{Z}, 4 j) \times \prod_{j=0}^{\infty} K\left(\mathbb{Z}_{2}, 4 j+2\right) \\
\mathcal{Z}_{\mathbb{H}}^{\text {odd }} & \cong \prod_{j=0}^{\infty} K(\mathbb{Z}, 4 j) \times \prod_{j=0}^{\infty} K\left(\mathbb{Z}_{2}, 4 j+1\right)
\end{aligned}
$$

Proof This corollary follows from Proposition 3.1 Theorem 2.3. and [15, Theorem A.5]. 
We now observe that the algebraic join gives biadditive maps

$$
\#: \mathcal{Z}_{\mathbb{H}}^{q}(\mathbb{P}(V)) \wedge \mathcal{Z}_{\mathbb{H}}^{q^{\prime}}\left(\mathbb{P}\left(V^{\prime}\right)\right) \longrightarrow \mathcal{Z}_{\mathbb{H}}^{q+q^{\prime}}\left(\mathbb{P}\left(V \oplus V^{\prime}\right)\right)
$$

for all quaternionic vector spaces $V, V^{\prime}$ and for all $q, q^{\prime}$. These maps induce pairings

$$
\pi_{k} \mathcal{Z}_{\mathbb{H}}^{q}(\mathbb{P}(V)) \otimes \pi_{k^{\prime}} \mathcal{Z}_{\mathbb{H}}^{q^{\prime}}\left(\mathbb{P}\left(V^{\prime}\right)\right) \longrightarrow \pi_{k+k^{\prime}} \mathcal{Z}_{\mathbb{H}}^{q+q^{\prime}}\left(\mathbb{P}\left(V \oplus V^{\prime}\right)\right)
$$

which together with Proposition 3.1 and Corollary 3.2 above gives us the following.

Proposition 3.3 Set $\mathfrak{R}=\mathfrak{R}_{*}^{0} \oplus \mathfrak{R}_{*}^{1}$, where

$$
\mathfrak{R}_{*}^{0}=\pi_{*} \mathcal{Z}_{\mathbb{H}}^{\text {ev }} \quad \text { and } \quad \mathfrak{R}_{*}^{1}=\pi_{*} \mathcal{Z}_{\mathbb{H}}^{\text {odd }} .
$$

Then the algebraic join gives $\mathfrak{R}$ the structure of a $\mathbb{Z}_{2} \times \mathbb{Z}$-graded ring.

The following determination of this ring will be established in Section 7

Theorem 3.4 The subring $\mathfrak{R}_{*}^{0}$ admits an isomorphism

$$
\mathfrak{R}_{*}^{0} \cong \mathbb{Z}[x, u] /\left(2 u, u^{2}\right)
$$

where $x$ corresponds to the generator of $\pi_{4} \mathcal{Z}_{\mathbb{H}}^{\mathrm{ev}} \cong \mathbb{Z}, u$ corresponds to the generator of $\pi_{2} \mathcal{Z}_{\mathbb{H}}^{\text {ev }} \cong \mathbb{Z}_{2}$, and $\left(2 u, u^{2}\right)$ denotes the ideal in the ring $\mathbb{Z}[x, u]$ generated by $2 u$ and $u^{2}$.

With respect to the isomorphism (44), one has that $u \cdot \mathfrak{R}_{*}^{1}=0$ and $\mathfrak{R}_{*}^{1}$ is the $\mathbb{Z}[x]$-module

$$
\mathfrak{R}_{*}^{1} \cong \mathbb{Z}[x] \lambda \oplus \mathbb{Z}_{2}[x] v
$$

where $\lambda$ corresponds to the generator of $\pi_{0} \mathfrak{R}_{*}^{1}=\mathbb{Z}$ and $v$ corresponds to the generator of $\pi_{1} \mathfrak{R}_{*}^{1}=\mathbb{Z}_{2}$.

The elements $\lambda$ and $v$ satisfy the relations

$$
\lambda^{2}=4, \quad \lambda \cdot v=0 \quad \text { and } \quad v^{2}=0 .
$$

Note 3.5 Note that $\mathfrak{R}_{*}$ is a $\mathbb{Z}_{2}$-graded $\mathbb{Z}[x]$-algebra with even generators 1 and $u$ and "companion" odd generators $\lambda$ and $v$. 


\section{Extending functors from $K$-theory}

As in the case of real cycles, certain basic functors from representation theory extend to quaternionic algebraic cycles. The constructions parallel those of the real case but curiously the roles are interchanged.

Quaternionification To any complex vector space $V$ we can associate the quaternionic vector space $\left(V \otimes_{\mathbb{C}} \mathbb{H}, \mathbf{j}\right)$ where

$$
\left(V \otimes_{\mathbb{C}} \mathbb{H}, \mathbf{j}\right) \stackrel{\text { def }}{=} V \oplus \bar{V} \text { and } \mathbf{j}(v, w)=(-w, v) .
$$

Recall that if $V$ is a complex vector space, we define $\bar{V}$ to be the same underlying real vector space with complex structure changed from $J$ to $-J$. For any $q<\operatorname{dim}(V)$ we have a map

$$
\mathcal{Z}_{\mathbb{C}}^{q}(\mathbb{P}(V)) \longrightarrow \mathcal{Z}_{\mathbb{H}}^{2 q}(\mathbb{P}(V \oplus \bar{V}))
$$

defined by

$$
c \mapsto c \# c .
$$

This construction gives rise to commutative diagrams

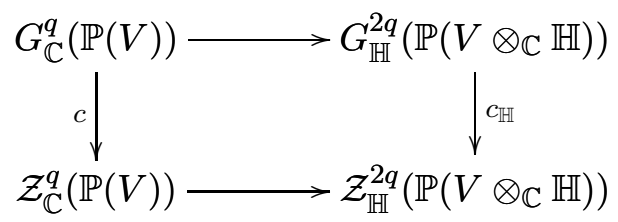

where $G_{\mathbb{H}}^{2 q}$ denotes the Grassmannian of quaternionic linear subspaces of quaternionic codimension $q$. Diagram (8) stabilizes to a commutative diagram

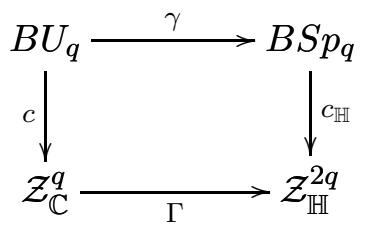

which in light of [13, Theorem 1], [15, Theorem A.5] and Theorem 2.3 can be rewritten canonically as

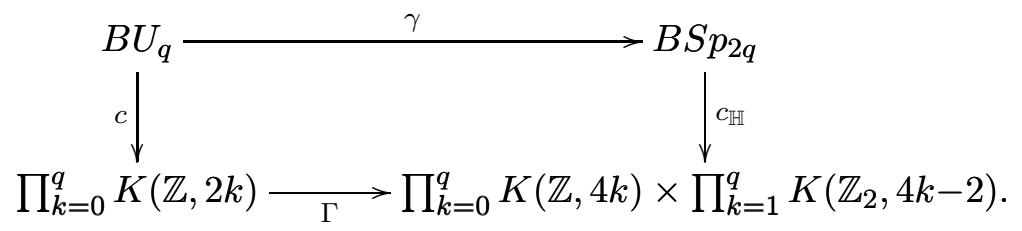


The forgetful functor Consider a quaternionic vector space $(V, \mathbf{j})$ and the functor $(V, \mathbf{j}) \mapsto V$ which forgets the quaternionic structure. For any $q<$ $\operatorname{dim}_{\mathbb{H}}(V)$ we have a map

$$
\mathcal{Z}_{\mathbb{H}}^{2 q}(\mathbb{P}(V)) \longrightarrow \mathcal{Z}_{\mathbb{C}}^{2 q}(\mathbb{P}(V))
$$

which simply includes the $\mathbf{j}$-fixed cycles into the group of all cycles. This gives commutative diagrams

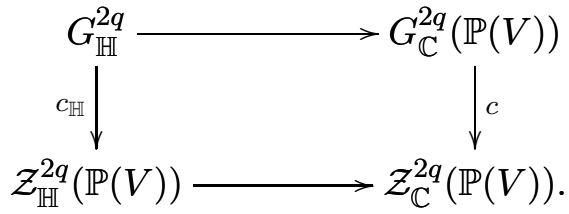

Note that $G_{\mathbb{H}}^{2 q}$ is exactly the subset of $\mathbf{j}$-fixed planes $G_{\mathbb{C}}^{2 q}$. The diagram (12) stabilizes to

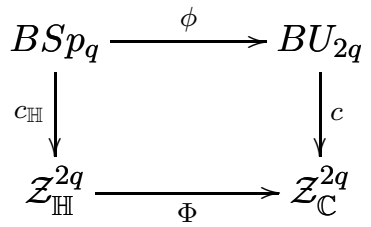

where $\phi: B S p_{q} \rightarrow B U_{2 q}$ is the map induced by the standard embedding $S p_{q} \rightarrow U_{2 q}$ given by forgetting the quaternionic structure. Diagram (13) can be rewritten as

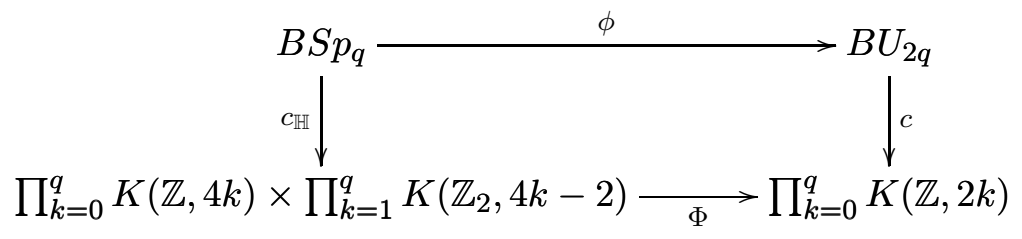

where these splittings are canonical; cf Theorem 2.3. [13, Theorem 1] and [15, Theorem A.5].

Let $\iota_{2 k} \in H^{2 k}(K(\mathbb{Z}, 2 k) ; \mathbb{Z})$ be the fundamental class as above, and denote by $\xi_{\mathbb{H}}^{q}$ the universal quaternionic bundle of quaternionic rank $q$ over $B S p_{q}$. Since $c^{*} \iota_{2 k}$ is the universal $k$ th Chern class, the commutativity of (14) shows that

$$
c_{\mathbb{H}}{ }^{*} \Phi^{*}\left(\iota_{2 k}\right)=\phi^{*} c_{k}\left(\xi_{\mathbb{C}}^{2 q}\right)=c_{k}\left(\xi_{\mathbb{H}}^{q}\right)= \begin{cases}\sigma_{m} & \text { if } k=2 m \text { and } 0 \leq m \leq q, \\ 0 & \text { otherwise }\end{cases}
$$

where $\sigma_{1}, \ldots, \sigma_{q}$ are the canonical generators of $H^{*}\left(B S p_{q} ; \mathbb{Z}\right)=\mathbb{Z}\left[\sigma_{1}, \ldots, \sigma_{q}\right]$.

The map $\Phi$ is entirely determined up to homotopy by the following result. 
Theorem 4.1 Let $\iota_{2 k} \in H^{2 k}(K(\mathbb{Z}, 2 k) ; \mathbb{Z})=\mathbb{Z}$ be the fundamental class. Then

$$
\Phi^{*} \iota_{4 k}=\iota_{4 k} \quad \text { and } \quad \Phi^{*} \iota_{4 k-2}=0
$$

for all $k$.

Proof The diagrams (14) successively embed one into the next for $q=1,2, \ldots$ by taking linear embeddings $\cdots \subset \mathbb{H}^{n} \subset \mathbb{H}^{n+1} \subset \ldots$ Passing to quotients and using Theorem 6.6 below gives

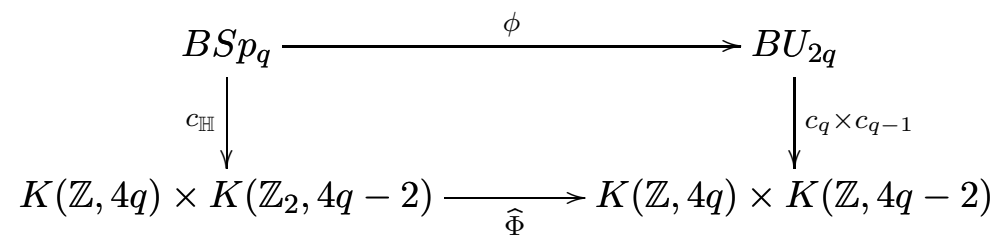

where $\widehat{\Phi}: \mathcal{Z}_{\mathbb{H}}^{2 q} / \mathcal{Z}_{\mathbb{H}}^{2 q-2} \longrightarrow \mathcal{Z}_{\mathbb{C}}^{2 q} / \mathcal{Z}_{\mathbb{C}}^{2 q-2}$ is the induced map of quotient groups. The second assertion of the theorem follows from the fact that

$$
H^{4 q-2}\left(K(\mathbb{Z}, 4 q) \times K\left(\mathbb{Z}_{2}, 4 q-2\right) ; \mathbb{Z}\right)=0 .
$$

Now

$$
\begin{aligned}
H^{4 q}(K(\mathbb{Z}, 4 q) & \left.\times K\left(\mathbb{Z}_{2}, 4 q-2\right) ; \mathbb{Z}\right) \\
& =H^{4 q}(K(\mathbb{Z}, 4 q) ; \mathbb{Z}) \oplus H^{4 q}\left(K\left(\mathbb{Z}_{2}, 4 q-2\right) ; \mathbb{Z}\right) \\
& =H^{4 q}(K(\mathbb{Z}, 4 q) ; \mathbb{Z})=\mathbb{Z} \iota_{4 q} .
\end{aligned}
$$

By (15) we see that $c_{\mathbb{H}}{ }^{*} \Phi^{*}\left(\iota_{2 k}\right)$ is an additive generator and therefore $\Phi^{*}\left(\iota_{2 k}\right)$ must be also. This proves the first assertion.

Relations Consider the diagram

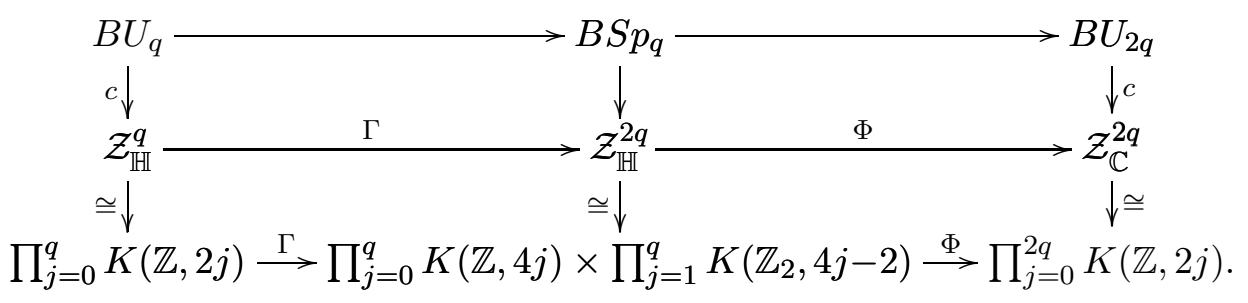

From (77) we see that if $V$ has a real structure $\rho$, then under the isomorphism $I \oplus \rho: V \oplus \bar{V} \longrightarrow V \oplus V$, the map $\Gamma: \mathcal{Z}_{\mathbb{C}}^{q} \rightarrow \mathcal{Z}_{\mathbb{H}}^{2 q}$ becomes $\Gamma(c)=c \# \rho_{*}(c)$. It follows that

$$
\Phi \circ \Gamma(c)=c \# \rho_{*}(c)
$$

for $c \in \mathcal{Z}_{\mathbb{C}}^{q}$. As in [15, Proposition 5.1] we conclude the following. 
Proposition 4.2 Let $\iota_{2 k} \in H^{2 k}(K(\mathbb{Z}, 2 k) ; \mathbb{Z})=\mathbb{Z}$ be the fundamental class. Then for each $k$ the composition $\Phi \circ \Gamma$ satisfies

$$
(\Phi \circ \Gamma)^{*} \iota_{2 k}=\sum_{i+j=k}(-1)^{j} \iota_{2 i} \cup \iota_{2 j}
$$

Note that in particular $(\Phi \circ \Gamma)^{*} \iota_{4 k-2}=0$ as predicted by Theorem 4.1

Combining Theorem 4.1 and Proposition 4.2 gives the following.

Corollary 4.3 For each $k \leq q$ one has

$$
\Gamma^{*} \iota_{4 k}=\sum_{\substack{i+j=2 k \\ i, j \leq q}}(-1)^{j} \iota_{2 i} \cup \iota_{2 j}+(-1)^{k} \iota_{2 k}^{2} .
$$

To completely determine $\Gamma$ up to homotopy we need to compute the classes $\Gamma^{*} \widetilde{\iota}_{4 k-2}$ where $\widetilde{\iota}_{4 k-2} \in H^{4 k-2}\left(K\left(\mathbb{Z}_{2}, 4 k-2\right) ; \mathbb{Z}_{2}\right)$ denotes the fundamental class. From the commutative diagram (10) we see that

$$
c^{*} \Gamma^{*} \widetilde{\iota}_{4 k-2}=\gamma^{*} c_{\mathbb{H}}^{*} \widetilde{\iota}_{4 k-2}=0
$$

since $H^{4 k-2}\left(B S p_{q} ; \mathbb{Z}_{2}\right)=0$. Thus $\Gamma^{*} \widetilde{\iota}_{4 k-2}$ lies in the kernel of $c^{*}$ on $\bmod 2$ cohomology. (Note that $c^{*}$ is injective on $\mathbb{Z}_{2}\left[\iota_{1}, \ldots, \iota_{q}\right]$.) However, a complete calculation of this class remains to be done.

\section{$5 \quad$ Infinite loop space structures.}

In this section we carry the discussion in Section 6 of [15] over to the quaternionic case. Given a quaternionic vector space $(V, \mathbf{j})$ with $\operatorname{dim}_{\mathbb{C}}(V)=2 q$, we define $\mathcal{I}_{*}$-functors

$$
T_{G_{\mathbb{H}}}(V)=G_{\mathbb{H}}^{2 q}\left(\mathbb{P}(V \oplus V) \quad \text { and } \quad T_{Z_{\mathbb{H}}}(V)=\mathcal{Z}_{\mathbb{H}}^{2 q}(\mathbb{P}(V \oplus V) .\right.
$$

The action on morphisms and the natural transformations $\omega_{G_{\mathbb{H}}}$ and $\omega_{Z_{\mathbb{H}}}$ are defined exactly as in [15, Section 6]. The inclusion

$$
T_{G_{\mathbb{H}}}(V) \subset T_{Z_{\mathbb{H}}}(V)
$$

as cycles of degree one is a natural transformation of $\mathcal{I}_{*}$-functors. As seen in [20. page 16], the limiting space

$$
\lim _{q \rightarrow \infty} T_{G_{\mathbb{H}}}\left(\mathbb{H}^{q}\right)=\mathbf{B S p}
$$

is a connected $\mathcal{L}$-space whose associated infinite loop space structure coincides with the usual connective Bott structure. The arguments of [15, Section 6] and 15. Theorems 6.9 and 6.10] apply directly to prove the following. 
Theorem 5.1 The limiting space $\mathcal{Z}_{\mathbb{H}}^{\mathrm{ev}}$ is an $E_{\infty}$-ring space and forms the 0 -level space of an $E_{\infty}$-ring spectrum. The component $\mathcal{Z}_{\mathbb{H}}^{\text {ev }}(1)$ consisting of cycles of degree 1 carries an infinite loop space structure which enhances the algebraic join and for which the induced mapping

$$
\mathbf{B S p} \longrightarrow \mathcal{Z}_{\mathbb{H}}^{e v}(1)
$$

is a map of infinite loop spaces.

Consider now the "forgetful" homomorphism

$$
\Phi: \mathcal{Z}_{\mathbb{H}}^{2 q}(\mathbb{P}(V \oplus V)) \longrightarrow \mathcal{Z}_{\mathbb{C}}^{2 q}(\mathbb{P}(V \oplus V))
$$

defined in the last section. This is a natural transformation of $\mathcal{I}_{*}$-functors, and so we have:

Proposition 5.2 The limiting "forgetful" homomorphism

$$
\Phi: \mathcal{Z}_{\mathbb{H}}^{\mathrm{ev}} \longrightarrow \mathcal{Z}_{\mathbb{C}}^{\infty}
$$

is a map of $E_{\infty}$ ring spaces. In particular, its restriction $\Phi: \mathcal{Z}_{\mathbb{H}}^{\text {ev }}(1) \longrightarrow \mathcal{Z}_{\mathbb{C}}^{\infty}(1)$ is an infinite loop map.

Now the "quaternionification" maps

$$
\Gamma: \mathcal{Z}_{\mathbb{C}}^{2 q}(\mathbb{P}(V \oplus V)) \longrightarrow \mathcal{Z}_{\mathbb{H}}^{4 q}(\mathbb{P}(V \oplus \bar{V} \oplus V \oplus \bar{V}))
$$

are not additive mappings. Nevertheless, they do give natural transformations of $\mathcal{I}_{*}$-functors (with values in topological spaces, not topological groups). Hence we have:

Proposition 5.3 The limiting "quaternionification" mapping

$$
\Gamma: \mathcal{Z}_{\mathbb{C}}^{\infty} \longrightarrow \mathcal{Z}_{\mathbb{H}}^{\mathrm{ev}}
$$

is a mapping of $\mathcal{L}$-spaces. In particular, its restriction $\Gamma$ : $\mathcal{Z}_{\mathbb{C}}^{\infty}(1) \longrightarrow \mathcal{Z}_{\mathbb{H}}^{\mathrm{ev}}(1)$ is an infinite loop map.

These maps sit in a commutative diagram:

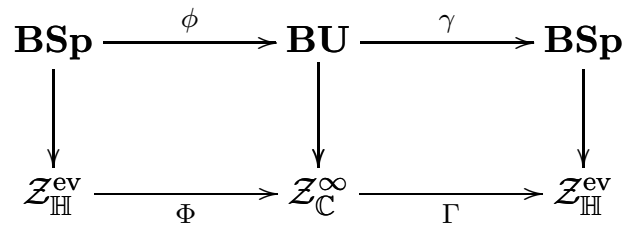




\section{Proof of Theorem 2.3}

Part (ii) of this result was established in [14, Theorem 6.4], so it remains only to prove part (i). The Quaternionic Suspension Theorem of [14, Theorem 6.1] applies also to cycles of even codimension, but since quaternionic suspension changes cycle-dimension by 2 , one is unable in this case to reduce to 0 -cycles where the Dold-Thom Theorem can be used. We shall solve this problem by "replacing" $\mathbb{P}(V)$ with an even-dimensional variety $Q(V)$.

To begin consider the Veronese embedding $v: \mathbb{P}(V) \hookrightarrow \mathbb{P}\left(\operatorname{Sym}^{2}(V)\right)$ which converts $\mathbb{P}(V)$ to a real subvariety under a real structure $r: \mathbb{P}\left(\operatorname{Sym}^{2}(V)\right) \rightarrow$ $\mathbb{P}\left(\operatorname{Sym}^{2}(V)\right)$ coming from a complex conjugation on $\operatorname{Sym}^{2}(V)$. (To see this explicitly choose coordinates $z_{\alpha}+w_{\alpha} j$, for $\alpha=1, \ldots, n$, on $V=\mathbb{H}^{n}$ and note that $v\left(z_{\alpha}, w_{\alpha}\right)=\left(z_{\alpha} z_{\beta}, w_{\alpha} w_{\beta}, z_{\alpha} w_{\beta}\right)$.) The following is a direct consequence of [12. See also 14].

Proposition 6.1 The $\mathbb{Z}_{2}$-equivariant complex suspension map

$$
\mathbb{Z}: \mathcal{Z}^{q}(\mathbb{P}(V)) \longrightarrow \mathcal{Z}^{q}(Q(V))
$$

where

$$
Q(V) \equiv \not ⿱\left\{\{v(\mathbb{P}(V))\}=\operatorname{Thom}\left\{\mathcal{O}_{\mathbb{P}(V)}(2)\right\}\right.
$$

is a $\mathbb{Z}_{2}$-homotopy equivalence.

The idea now is to compute the $\mathbb{Z}_{2}$-homotopy type of the spaces $\mathcal{Z}^{q}(Q(V))$, for $q$ even, by "de-suspending" to the case of 0 -cycles. To begin we fix some notation. Let

$$
\mathcal{Q}^{2 n} \equiv Q\left(\mathbb{H}^{n}\right)-\{\infty\} \equiv \mathcal{O}_{\mathbb{P}_{\mathbb{C}}\left(\mathbb{H}^{n}\right)}(2) \stackrel{p}{\rightarrow} \mathbb{P}_{\mathbb{C}}\left(\mathbb{H}^{n}\right)
$$

denote the square of the complex hyperplane bundle over $\mathbb{P}_{\mathbb{C}}\left(\mathbb{H}^{n}\right)$. Our real structure, $r: Q\left(\mathbb{H}^{n}\right) \longrightarrow Q\left(\mathbb{H}^{n}\right)$, when restricted to $\mathcal{Q}^{2 n}$, is an anti-linear bundle map for which the diagram

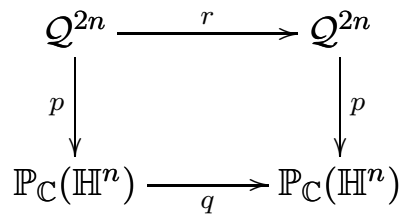

commutes. (This bundle map is the one naturally induced on $\mathcal{O}(2)$ via multiplication by the quaternion $j$.) Now the topological groups of algebraic cycles, $\mathcal{Z}^{q}(U)$ are defined for any quasi-projective variety $U$ (cf [17, Definition 4.5]), 
and, since $Q\left(\mathbb{H}^{n}\right)-\mathcal{Q}^{2 n}$ consists of a single point, there are $\mathbb{Z}_{2}$-equivariant homeomorphisms

$$
\mathcal{Z}^{q}\left(\mathcal{Q}^{2 n}\right) \cong \begin{cases}\mathcal{Z}^{q}\left(Q\left(\mathbb{H}^{n}\right)\right) & \text { for } q<2 n, \\ \mathcal{Z}^{q}\left(Q\left(\mathbb{H}^{n}\right)\right) / \mathbb{Z} & \text { for } q=2 n .\end{cases}
$$

We now observe that there is a commutative diagram of $\mathbb{Z}_{2}$-equivariant bundle maps

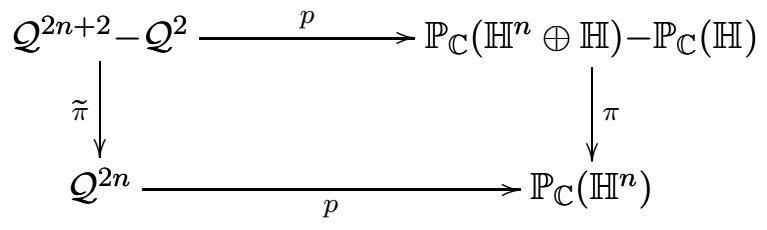

where $\pi$ is linear projection and $\tilde{\pi}$ is defined as follows. Let $\ell_{0} \longrightarrow \mathbb{P}_{\mathbb{C}}\left(\mathbb{H}^{n}\right)$ and $\ell \longrightarrow \mathbb{P}_{\mathbb{C}}\left(\mathbb{H}^{n} \oplus \mathbb{H}\right)$ denote the tautological complex line bundles $\mathcal{O}(-1)$, and note that

$$
\mathcal{Q}^{2 n+2}=\operatorname{Hom}(\ell \otimes \ell, \mathbb{C}) \quad \text { and } \quad \mathcal{Q}^{2 n}=\operatorname{Hom}\left(\ell_{0} \otimes \ell_{0}, \mathbb{C}\right) .
$$

The linear projection $\mathbb{H}^{n} \oplus \mathbb{H} \longrightarrow \mathbb{H}^{n}$ induces a bundle mapping $\pi_{*}: \ell \longrightarrow \ell_{0}$ covering $\pi$ which is an isomorphism on fibres. The map $\tilde{\pi}$ in (22) is given by $\tilde{\pi}(h)=h \circ\left(\pi_{*}^{-1} \otimes \pi_{*}^{-1}\right)$ for $h \in \operatorname{Hom}(\ell \otimes \ell, \mathbb{C})$.

Our main assertion here is the following.

Proposition 6.2 The flat pull-back of cycles gives a $\mathbb{Z}_{2}$-homotopy equivalence

$$
\tilde{\pi}^{*}: \mathcal{Z}^{q}\left(\mathcal{Q}^{2 n}\right) \longrightarrow \mathcal{Z}^{q}\left(\mathcal{Q}^{2 n+2}-\mathcal{Q}^{2}\right)
$$

for all $q \leq 2 n$.

Interesting note 6.3 A quick proof of Proposition 6.2 can be given for $q<2 n$ as follows. By [14] and 12] the flat pull-back of cycles gives equivariant homotopy equivalences

$$
\begin{aligned}
\pi^{*}: \mathcal{Z}^{q}\left(\mathbb{P}_{\mathbb{C}}\left(\mathbb{H}^{n}\right)\right) & \longrightarrow \mathcal{Z}^{q}\left(\mathbb{P}_{\mathbb{C}}\left(\mathbb{H}^{n} \oplus \mathbb{H}\right)-\mathbb{P}_{\mathbb{C}}(\mathbb{H})\right) \cong \mathcal{Z}^{q}\left(\mathbb{P}_{\mathbb{C}}\left(\mathbb{H}^{n+1}\right)\right) \\
\text { and } \quad p^{*}: \mathcal{Z}^{q}\left(\mathbb{P}_{\mathbb{C}}\left(\mathbb{H}^{n}\right)\right) & \longrightarrow \mathcal{Z}^{q}\left(\mathcal{Q}^{2 n}\right)
\end{aligned}
$$

for all $q$ and $n$; see also [4, Proposition 4.15]. Equivariant excision arguments (cf [17, 18, 14] or 4, Remark 4.14]) then show that

$$
p^{*}: \mathcal{Z}^{q}\left(\mathbb{P}_{\mathbb{C}}\left(\mathbb{H}^{n} \oplus \mathbb{H}\right)-\mathbb{P}_{\mathbb{C}}(\mathbb{H})\right) \longrightarrow \mathcal{Z}^{q}\left(\mathcal{Q}^{2 n+2}-\mathcal{Q}^{2}\right)
$$

is also a $\mathbb{Z}_{2}$-homotopy equivalence, and the Proposition follows from the commutativity of (22). Unfortunately this will not allow us to reduce to 0 -cycles, so we must construct a proof directly in this case. 
Proof of Proposition 6.2 We compactify each $\mathcal{Q}^{2 n} \cong \mathcal{O}_{\mathbb{P}_{\mathbb{C}}\left(\mathbb{H}^{n}\right)}(2)$ by taking the projective closure

$$
\overline{\mathcal{Q}}^{2 n}=\mathbb{P}\{\mathcal{O}(2) \oplus \mathbb{C}\} .
$$

This gives us a fibre square

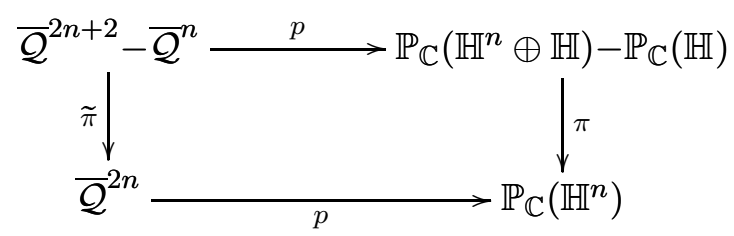

of smooth $\mathbb{Z}_{2}$-maps. We recover the diagram (22) by removing the restriction of $\tilde{\pi}$ to the " $\infty$-section" $\mathbb{P}_{\infty}^{2 n-1} \subset \overline{\mathcal{Q}}^{2 n}$. Observe that taking the graph gives an equivariant isomorphism

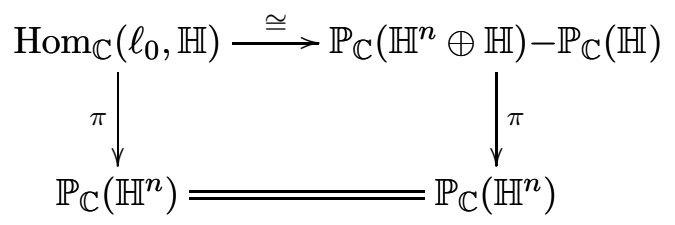

where the $\mathbb{Z}_{2}$-action on $\operatorname{Hom}_{\mathbb{C}}\left(\ell_{0}, \mathbb{H}\right)$ is given by sending a linear map $h: \ell_{0} \longrightarrow$ $\mathbb{H}$ to $j(h)=j \circ h \circ j^{-1}$. Via this isomorphism we can rewrite the fibre square in (23) as a pull-back diagram

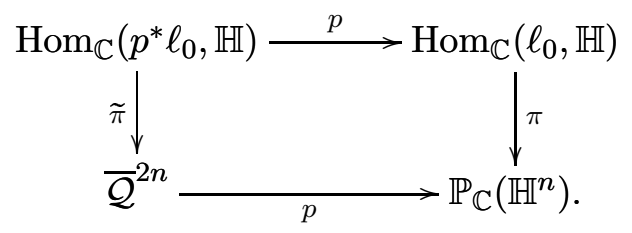

The restriction

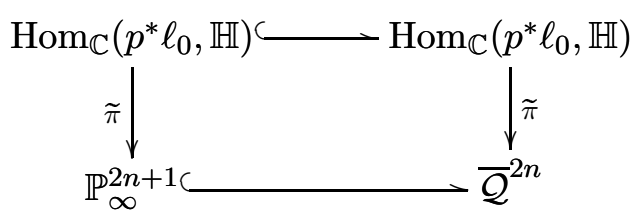

of $\widetilde{\pi}$ to the infinity-section is isomorphic (via $p$ ) to the bundle

$$
\mathbb{P}_{\mathbb{C}}\left(\mathbb{H}^{n} \oplus \mathbb{H}\right)-\mathbb{P}_{\mathbb{C}}(\mathbb{H}) \longrightarrow \mathbb{P}_{\mathbb{C}}\left(\mathbb{H}^{n}\right)
$$

for which the Quaternionic Suspension Theorem holds. Thus by equivariant excision (see [4, Remark 4.14]) our Proposition will follow if we can prove that

$$
\tilde{\pi}^{*}: \mathcal{Z}^{q}\left(\overline{\mathcal{Q}}^{2 n}\right) \longrightarrow \mathcal{Z}^{q}\left(\operatorname{Hom}_{\mathbb{C}}\left(p^{*} \ell_{0}, \mathbb{H}\right)\right)
$$


is a $\mathbb{Z}_{2}$-homotopy equivalence for all $q$. For our application the interesting case (and by Note 6.3 the only remaining case) is where $q=2 n$. Thus we shall prove the assertion that

$$
\tilde{\pi}^{*}: \mathcal{Z}_{0}\left(\overline{\mathcal{Q}}^{2 n}\right) \longrightarrow \mathcal{Z}_{2}\left(\operatorname{Hom}_{\mathbb{C}}\left(p^{*} \ell_{0}, \mathbb{H}\right)\right) \text { is a } \mathbb{Z}_{2} \text {-homotopy equivalence }
$$

where $\mathcal{Z}_{p}$ denotes the group of cycles of dimension $p$.

To prove (26) we consider the submonoid

$$
\mathcal{T}_{2}^{+} \subset \mathcal{C}_{2}\left(\operatorname{Hom}_{\mathbb{C}}\left(p^{*} \ell_{0}, \mathbb{H}\right)\right)
$$

of effective 2-cycles which meet the zero-section in proper dimension (namely 0 ), and denote by

$$
\mathcal{T}_{2} \subset \mathcal{Z}_{2}\left(\operatorname{Hom}_{\mathbb{C}}\left(p^{*} \ell_{0}, \mathbb{H}\right)\right)
$$

the induced homomorphism of naïve topological group completions.

Observe now that scalar multiplication

$$
\Phi_{t}: \operatorname{Hom}_{\mathbb{C}}\left(p^{*} \ell_{0}, \mathbb{H}\right) \longrightarrow \operatorname{Hom}_{\mathbb{C}}\left(p^{*} \ell_{0}, \mathbb{H}\right)
$$

by real numbers $t>0$ gives bundle maps commuting with the $\mathbb{Z}_{2}$-action, and pulling to the normal cone gives a $\mathbb{Z}_{2}$-deformation retraction

$$
\mathcal{T}_{2} \longrightarrow \tilde{\pi}^{*}\left\{\mathcal{Z}_{0}\left(\overline{\mathcal{Q}}^{n}\right)\right\}
$$

(cf [14, Assertion 1, Section 2.3], 9], 13]). Therefore, it remains only to show that the inclusion (28) is a $\mathbb{Z}_{2}$-homotopy equivalence.

We shall proceed in analogy with the arguments in [14, Section 2.3]. We consider the direct sum

$$
\operatorname{Hom}_{\mathbb{C}}\left(p^{*} \ell_{0}, \mathbb{H}\right) \oplus \operatorname{Hom}_{\mathbb{C}}\left(p^{*} \ell_{0}, \mathbb{H}\right) \longrightarrow \overline{\mathcal{Q}}^{n}
$$

and choose two distinct projections

$$
\pi_{0}, \pi_{\infty}: \operatorname{Hom}_{\mathbb{C}}\left(p^{*} \ell_{0}, \mathbb{H}\right) \oplus \operatorname{Hom}_{\mathbb{C}}\left(p^{*} \ell_{0}, \mathbb{H}\right) \longrightarrow \operatorname{Hom}_{\mathbb{C}}\left(p^{*} \ell_{0}, \mathbb{H}\right)
$$

The map $\pi_{0}$ is simply projection onto the first factor, and $\pi_{\infty}=\pi_{0} \circ S$ where

$$
S=\left(\begin{array}{cc}
\text { Id } & \epsilon J \\
0 & \text { Id }
\end{array}\right)
$$

$\epsilon>0$, and $J:\{0\} \oplus \operatorname{Hom}_{\mathbb{C}}\left(p^{*} \ell_{0}, \mathbb{H}\right) \stackrel{\cong}{\longrightarrow} \operatorname{Hom}_{\mathbb{C}}\left(p^{*} \ell_{0}, \mathbb{H}\right) \oplus\{0\}$ is the canonical isomorphism between the second and first factors. 
These projections can be viewed alternatively as follows. Via (24) and (25) we obtain a pull-back diagram

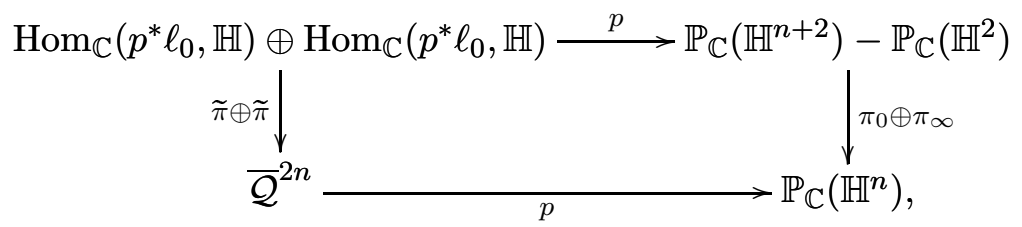

and $\pi_{0}, \pi_{\infty}$ are just the pull-backs of the ones constructed in $\mathbb{P}_{\mathbb{C}}\left(\mathbb{H}^{n+2}\right)$ by projecting away from quaternionic lines $\lambda_{0}, \lambda_{\infty} \subset \mathbb{P}_{\mathbb{C}}\left(\mathbb{H}^{2}\right)$.

Consider now the open dense set $\mathcal{U}(d)$ of divisors $D$ of degree $d$ on $\mathbb{P}_{\mathbb{C}}\left(\mathbb{H}^{n+2}\right)$ with the property that $D$ meets $j D$ in proper dimension and that $\mathbb{D}=D \bullet j D$ (and all scalar multiples $t \mathbb{D}$ for $0<t \leq 1$ ) do not meet the vertices $\lambda_{0}, \lambda_{\infty}$ of our projections To each $D \in \mathcal{U}(d)$ we associate the pull-back cycle $\widetilde{\mathbb{D}}=p^{*} \mathbb{D}$ and define a transformation

$$
\Psi_{D}: \mathcal{C}_{2}\left(\operatorname{Hom}_{\mathbb{C}}\left(p^{*} \ell_{0}, \mathbb{H}\right)\right) \longrightarrow \mathcal{C}_{2}\left(\operatorname{Hom}_{\mathbb{C}}\left(p^{*} \ell_{0}, \mathbb{H}\right)\right)
$$

as in [14, (2.6.1)], [13, page 285] by setting

$$
\Psi_{D}(c)=\left(\pi_{\infty}\right) *\left\{\pi_{0}^{*} c \bullet \widetilde{\mathbb{D}}\right\} .
$$

Note that $\pi_{\infty}$ and $\pi_{0}$ are proper on $\widetilde{\mathbb{D}}$. Note also that if $\operatorname{deg}(D)=d$, then $\lim _{t \rightarrow 0} \Psi_{t D}=d^{2} \cdot \mathrm{Id}$.

The arguments given in [14, pp 634-641] and the proof of [14, Theorem 6.1] may now be repeated in this context. The important point is to show that there is a function $N(d)$ such that $N(d) \rightarrow \infty$ as $d \rightarrow \infty$ and with the property that for any irreducible subvariety $Z \subset \operatorname{Hom}_{\mathbb{C}}\left(p^{*} \ell_{0}, \mathbb{H}\right)$ of dimension 4 ,

$$
\operatorname{codim}\left(\mathfrak{B}_{Z}(d)\right) \geq N(d)
$$

where

$$
\mathfrak{B}_{Z}(d)=\{D \in \mathcal{U}(d): \operatorname{dim}(Z \cap \widetilde{\mathbb{D}}) \geq 3\}
$$

is the set of "bad" divisors of degree $d$ for $Z$.

Now given such a $Z$, consider the irreducible subvariety $p(Z) \subset \mathbb{P}_{\mathbb{C}}\left(\mathbb{H}^{n+2}\right)$. Note that

$$
\operatorname{dim}(p(Z))=\text { either } 3 \text { or } 4
$$

and that the fibres of $p: Z \longrightarrow p(Z)$ are of dimension at most 1 since the fibres of $p$ are complex lines. 
Suppose that $\operatorname{dim}(p(Z))=3$. In [14, Lemma 2.7] it is proved that, since the $\mathbb{Z}_{2}$-action is free, there is a function $N(d)$ independent of $Z$ and going to infinity with $d$ such that

$$
\operatorname{codim}\{D \in \mathcal{U}(d): \operatorname{dim}(p(Z) \cap \mathbb{D}) \geq 2\} \geq N(d) .
$$

Since the fibre-dimension of $p$ is $\leq 1$, we see that

$$
\operatorname{dim}(p(Z) \cap \mathbb{D})=1 \quad \Rightarrow \quad \operatorname{dim}(Z \cap \widetilde{\mathbb{D}}) \leq 2
$$

and so the set of divisors in (29) contains $\mathfrak{B}_{Z}(d)$.

Suppose now that $\operatorname{dim}(p(Z))=4$. Then the generic fibre of $p: Z \longrightarrow p(Z)$ has dimension 0 , and there is a subvariety $\Sigma \subset p(Z)$ of dimension $\leq 3$ where the fibre dimension is 1. Again from [14, Section 2.7] we know that there is a function $N(d)$ as above such that

$$
\operatorname{codim}\{D \in \mathcal{U}(d): \operatorname{dim}(p(Z) \cap \mathbb{D}) \geq 3, \text { or } \operatorname{dim}(\Sigma \cap \mathbb{D}) \geq 1\} \geq N(d) .
$$

The set of divisors in (30) contains $\mathfrak{B}_{Z}(d)$, and so we have proved the desired estimate on the codimension of the bad sets. The arguments of [14, pp 640641] carry through to establish assertion (26), thereby completing the proof of Proposition 6.2

Corollary 6.4 There are $\mathbb{Z}_{2}$-homotopy equivalences

$$
\mathcal{Z}^{q}(Q(V)) \cong \mathcal{Z}^{q}(Q(V \oplus \mathbb{H}))
$$

for all $q \leq \operatorname{dim}_{\mathbb{C}}(V)$.

Proof Note that if $q<2 n$, then by (21)

$$
\begin{aligned}
\mathcal{Z}^{q}\left(Q\left(\mathbb{H}^{n}\right)\right) & =\mathcal{Z}^{q}\left(\mathcal{Q}^{2 n}\right) \cong \mathcal{Z}^{q}\left(\mathcal{Q}^{2 n+2}-\mathcal{Q}^{2}\right) \stackrel{\text { def }}{=} \mathcal{Z}^{q}\left(\mathcal{Q}^{2 n+2}\right) / \mathcal{Z}_{2 n+2-q}\left(\mathcal{Q}^{2}\right) \\
& =\mathcal{Z}^{q}\left(\mathcal{Q}^{2 n+2}\right)=\mathcal{Z}^{q}\left(Q\left(\mathbb{H}^{n+1}\right)\right)
\end{aligned}
$$

This case follows also from (19) and [14]. For the final case note that

$$
\begin{aligned}
\mathcal{Z}^{2 q}\left(Q\left(\mathbb{H}^{q}\right)\right) / \mathbb{Z} & =\mathcal{Z}^{2 q}\left(\mathcal{Q}^{2 q}\right) \cong \mathcal{Z}^{2 q}\left(\mathcal{Q}^{2 q+2}-\mathcal{Q}^{2}\right)=\mathcal{Z}^{2 q}\left(\mathcal{Q}^{2 q+2}\right) / \mathcal{Z}_{2}\left(\mathcal{Q}^{2}\right) \\
& =\mathcal{Z}^{2 q}\left(\mathcal{Q}^{2 q+2}\right) / \mathbb{Z}=\mathcal{Z}^{2 q}\left(Q\left(\mathbb{H}^{q+1}\right)\right) / \mathbb{Z}
\end{aligned}
$$

From (19) and Corollary 6.4 we conclude that there is a $\mathbb{Z}_{2}-$ homotopy equivalence

$$
\mathcal{Z}^{2 q}\left(\mathbb{P}_{\mathbb{C}}\left(\mathbb{H}^{n}\right)\right) \cong \mathcal{Z}_{0}\left(Q\left(\mathbb{H}^{q}\right)\right)
$$

and therefore there is a homotopy equivalence

$$
\mathcal{Z}_{\mathbb{H}}^{2 q}\left(\mathbb{P}_{\mathbb{C}}\left(\mathbb{H}^{n}\right)\right) \cong \mathcal{Z}_{0}\left(Q\left(\mathbb{H}^{q}\right)\right)^{\text {fixed }}
$$


Since $\mathbb{Z}_{2}$ acts freely outside of one point on $Q\left(\mathbb{H}^{q}\right)$, there is a group isomorphism

$$
\mathcal{Z}_{0}\left(Q\left(\mathbb{H}^{q}\right)\right)^{\text {fixed }} / \mathcal{Z}_{0}\left(Q\left(\mathbb{H}^{q}\right)\right)^{\text {av }}=\mathbb{Z}_{2}
$$

from which it follows that $\pi_{*}\left(\mathcal{Z}_{0}\left(Q\left(\mathbb{H}^{q}\right)\right)^{\text {fixed }}\right)=\pi_{*}\left(\mathcal{Z}_{0}\left(Q\left(\mathbb{H}^{q}\right)\right)^{\text {av }}\right)$. We also have that

$$
\mathcal{Z}_{0}\left(Q\left(\mathbb{H}^{q}\right)\right)^{\text {av }}=\mathcal{Z}_{0}\left(Q\left(\mathbb{H}^{q}\right) / \mathbb{Z}_{2}\right) .
$$

Now by the work of Dold-Thom [3] we know that for a connected finite complex $A$ there is a homotopy equivalence

$$
\mathcal{Z}_{0}(A) \cong \prod_{j \geq 0} K\left(H_{j}(A ; \mathbb{Z}), j\right)
$$

Therefore the first part of Theorem 2.3 (i) follows from the next Proposition.

\section{Proposition 6.5}

$$
H_{j}\left(Q\left(\mathbb{H}^{q}\right) / \mathbb{Z}_{2} ; \mathbb{Z}\right)= \begin{cases}\mathbb{Z} & \text { if } j \equiv 0(\bmod 4) \text { and } j \leq 4 q, \\ \mathbb{Z}_{2} & \text { if } j \equiv 2(\bmod 4) \text { and } j \leq 4 q-2, \\ 0 & \text { otherwise. }\end{cases}
$$

Proof Set $Y=Q\left(\mathbb{H}^{q}\right) / \mathbb{Z}_{2}$ and $X=\mathbb{P}_{\mathbb{C}}\left(\mathbb{H}^{q}\right) / \mathbb{Z}_{2}$, and note that $Y$ is the Thom space of a nonorientable real 2-plane bundle $L \rightarrow X$. ( $L$ is simply the quotient of $\mathcal{Q}^{2 q}=\mathcal{O}_{\mathbb{P}^{2 q-1}}(2)$ by the quaternion involution.) Thus,

$$
H_{*}(Y ; \mathbb{Z})=H_{*}\left(B_{L}, S_{L} ; \mathbb{Z}\right)
$$

where $S_{L} \subset B_{L}$ denote the unit circle and unit disk bundles of $L$. By looking at the Hopf fibration one can see that

$$
S_{L}=S^{4 q-1} / \mathbb{Z}_{4}
$$

where $\mathbb{Z}_{4}$ is generated by multiplication by the quaternion $j$ on the unit sphere $S^{4 q-1} \subset \mathbb{H}^{q}$. We know that:

$$
\begin{aligned}
H_{j}(X ; \mathbb{Z})= \begin{cases}\mathbb{Z} & \text { if } j \equiv 0(\bmod 4) \text { and } j \leq 4 q-4, \\
\mathbb{Z}_{2} & \text { if } j \equiv 1(\bmod 4) \text { and } j \leq 4 q-3, \\
0 & \text { otherwise }\end{cases} \\
H_{j}\left(S_{L} ; \mathbb{Z}\right)= \begin{cases}\mathbb{Z} & \text { if } j=0 \text { or } 4 q-1, \\
\mathbb{Z}_{4} & \text { if } j \text { is odd and } j<4 q-1, \\
0 & \text { otherwise }\end{cases}
\end{aligned}
$$


The long exact sequence in homology for the pair $\left(B_{L}, S_{L}\right)$ gives $H_{4 q}(Y ; \mathbb{Z})=$ $\mathbb{Z}$, and for $i<2 q$ it gives exact sequences

$$
0 \rightarrow H_{2 i}(X) \rightarrow H_{2 i}(Y) \rightarrow \mathbb{Z}_{4} \rightarrow H_{2 i-1}(X) \rightarrow H_{2 i-1}(Y) \rightarrow 0
$$

When $i=2 k$ we get

$$
0 \rightarrow \mathbb{Z} \rightarrow H_{4 k}(Y) \rightarrow \mathbb{Z}_{4} \rightarrow 0 \rightarrow H_{4 k-1}(Y) \rightarrow 0,
$$

and so $H_{4 k-1}(Y)=0$ and we have the short exact sequence

$$
0 \longrightarrow \mathbb{Z} \longrightarrow H_{4 k}(Y) \longrightarrow \mathbb{Z}_{4} \longrightarrow 0
$$

To understand this extension we consider the $\mathbb{Z}_{2}$-homology groups

$$
H_{j}\left(X ; \mathbb{Z}_{2}\right)= \begin{cases}0 & \text { if } j \equiv 3(\bmod 4) \text { and } j \leq 4 q-2 \\ \mathbb{Z}_{2} & \text { otherwise }\end{cases}
$$

By the Thom isomorphism,

$$
H_{j+2}\left(Y ; \mathbb{Z}_{2}\right) \cong H_{j}\left(X ; \mathbb{Z}_{2}\right)
$$

for all $j$. In particular we have $H^{4 k}\left(Y ; \mathbb{Z}_{2}\right)=H_{4 k}\left(Y ; \mathbb{Z}_{2}\right)=\mathbb{Z}_{2}$. We conclude that

$$
H_{4 k}(Y ; \mathbb{Z})=\mathbb{Z}
$$

since all other possibilities for the extension (34) force the dimension of the vector space $H^{4 k}\left(Y ; \mathbb{Z}_{2}\right)$ to be greater than 1 .

Now when $i=2 k+2$ in (33) we have

$$
0 \rightarrow H_{4 k+2}(Y) \rightarrow \mathbb{Z}_{4} \stackrel{\sigma}{\rightarrow} \mathbb{Z}_{2} \rightarrow H_{4 k+1}(Y) \rightarrow 0,
$$

and it remains to show that $\sigma \neq 0$. For this we must also consider the $\mathbb{Z}_{2}-$ homology groups

$$
H_{j}\left(S_{L} ; \mathbb{Z}_{2}\right)= \begin{cases}0 & \text { if } j>4 q-1, \\ \mathbb{Z}_{2} & \text { otherwise. }\end{cases}
$$

From the pair $\left(B_{L}, S_{L}\right)$ we get the exact sequence of $\mathbb{Z}_{2}$-homology groups

$$
\begin{aligned}
& 0 \longrightarrow H_{4 k+3}(Y) \longrightarrow H_{4 k+2}\left(S_{L}\right) \longrightarrow H_{4 k+2}(X) \longrightarrow \\
& \longrightarrow H_{4 k+2}(Y) \longrightarrow H_{4 k+1}\left(S_{L}\right) \longrightarrow H_{4 k+1}(X) \longrightarrow 0
\end{aligned}
$$

which becomes

$$
0 \rightarrow \mathbb{Z}_{2} \stackrel{\cong}{\rightarrow} \mathbb{Z}_{2} \stackrel{0}{\rightarrow} \mathbb{Z}_{2} \stackrel{\cong}{\rightarrow} \mathbb{Z}_{2} \stackrel{0}{\rightarrow} \mathbb{Z}_{2} \stackrel{\cong}{\rightarrow} \mathbb{Z}_{2} \rightarrow 0
$$

The generator of $H_{4 k+1}\left(S_{L} ; \mathbb{Z}\right)=\mathbb{Z}_{4}$ goes to the generator of $H_{4 k+1}\left(S_{L} ; \mathbb{Z}_{2}\right)=$ $\mathbb{Z}_{2}$, and from the line above we see that

$$
H_{4 k+1}\left(S_{L} ; \mathbb{Z}_{2}\right) \stackrel{\sigma \otimes \mathbb{Z}_{2}}{\longrightarrow} H_{4 k+1}\left(X ; \mathbb{Z}_{2}\right)
$$

is not zero. Hence, $\sigma$ is not zero, and the proposition is proved. 
We now observe that all the constructions in the proofs of Propositions 6.1 and 6.2 preserve the subgroups of averaged cycles, and so the suspension maps induce homotopy equivalences of these subgroups. Therefore, the suspension maps induce homotopy equivalences of the quotients

$$
\mathcal{Z}^{2 q}\left(Q\left(\mathbb{H}^{n}\right)\right)^{\text {fixed }} / \mathcal{Z}^{2 q}\left(Q\left(\mathbb{H}^{n}\right)\right)^{\text {av }} \cong \mathcal{Z}_{0}\left(Q\left(\mathbb{H}^{q}\right)\right)^{\text {fixed }} / \mathcal{Z}_{0}\left(Q\left(\mathbb{H}^{q}\right)\right)^{\text {av }}
$$

and by (32) the right hand side is the space of two points. This gives the second half of Theorem 2.3(i).

The canonical nature of the homotopy equivalences in Theorem 2.3 is established in [15, Appendix A].

From the argument above we can deduce the following.

Theorem 6.6 Fix $q<n$. Let

$$
\mathcal{Z}_{\mathbb{H}}^{2 q-2}\left(\mathbb{P}_{\mathbb{C}}\left(\mathbb{H}^{n-1}\right)\right) \subset \mathcal{Z}_{\mathbb{H}}^{2 q}\left(\mathbb{P}_{\mathbb{C}}\left(\mathbb{H}^{n}\right)\right)
$$

be the subgroup of cycles contained in the linear subspace $\mathbb{P}_{\mathbb{C}}\left(\mathbb{H}^{n-1}\right)$, and let

$$
\mathcal{Z}_{\mathbb{H}}^{2 q-1}\left(\mathbb{P}_{\mathbb{C}}\left(\mathbb{H}^{n-1}\right)\right) \subset \mathcal{Z}_{\mathbb{H}}^{2 q+1}\left(\mathbb{P}_{\mathbb{C}}\left(\mathbb{H}^{n}\right)\right)
$$

be defined similarly. Then the inclusion (35) is $(4 q-3)$-connected, and (36) is $(4 q-1)$-connected. Furthermore, there are canonical homotopy equivalences

$$
\begin{aligned}
\mathcal{Z}_{\mathbb{H}}^{2 q} / \mathcal{Z}_{\mathbb{H}}^{2 q-2} & \cong K(\mathbb{Z}, 4 q) \times K\left(\mathbb{Z}_{2}, 4 q-2\right), \\
\mathcal{Z}_{\mathbb{H}}^{2 q+1} / \mathcal{Z}_{\mathbb{H}}^{2 q-1} & \cong K(\mathbb{Z}, 4 q) \times K\left(\mathbb{Z}_{2}, 4 q-1\right) .
\end{aligned}
$$

Proof As in the proof of [15, Proposition 8.1] we see that the short exact sequence

$$
0 \longrightarrow \mathcal{Z}_{\mathbb{H}}^{2 q-1} \longrightarrow \mathcal{Z}_{\mathbb{H}}^{2 q+1} \longrightarrow \mathcal{Z}_{\mathbb{H}}^{2 q+1} / \mathcal{Z}_{\mathbb{H}}^{2 q-1} \longrightarrow 0
$$

is a fibration sequence. Quaternionic algebraic suspension [14, Theorem 6.1] shows that this sequence is equivalent to the fibration sequence

$$
0 \longrightarrow \mathcal{Z}_{0}\left(\mathbb{P}_{\mathbb{C}}^{2 q-1} / \mathbb{Z}_{2}\right) \stackrel{i_{*}}{\longrightarrow} \mathcal{Z}_{0}\left(\mathbb{P}_{\mathbb{C}}^{2 q+1} / \mathbb{Z}_{2}\right) \longrightarrow \mathcal{Z}_{0}\left(\left(\mathbb{P}_{\mathbb{C}}^{2 q+1} / \mathbb{P}_{\mathbb{C}}^{2 q-1}\right) / \mathbb{Z}_{2}\right) \longrightarrow 0 .
$$

One sees directly that $i: \mathbb{P}_{\mathbb{C}}^{2 q-1} / \mathbb{Z}_{2} \subset \mathbb{P}_{\mathbb{C}}^{2 q+1} / \mathbb{Z}_{2}$ induces an isomorphism of $H_{k}(\bullet ; \mathbb{Z})$ for all $k \leq 4 q-1$. Hence by the Dold-Thom Theorem $i_{*}$ induces an isomorphism of $\pi_{k}(\bullet)$ for $k$ in the same range. This proves the theorem for $\mathcal{Z}_{\mathbb{H}}^{2 q+1}$. The result for $\mathcal{Z}_{\mathbb{H}}^{2 q}$ is proved analogously using the suspension arguments above. 


\section{Proof of Theorem 3.4}

We begin by observing that for quaternionic vector spaces $V, V^{\prime}$ there is a commutative diagram

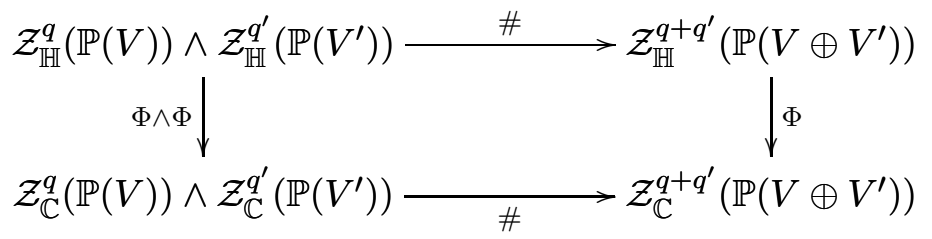

where the vertical maps $\Phi$ are given by the simple inclusion of the quaternionic cycles into the group of all cycles (cf Section 4). Under stabilization these maps yield a ring homomorphism

$$
\Phi_{*}: \mathfrak{R}_{*} \longrightarrow \pi_{*} \mathcal{Z}_{\mathbb{C}}^{\infty}=\mathbb{Z}[s] .
$$

Proposition 7.1 Let $p: \mathbb{P}_{\mathbb{C}}\left(\mathbb{H}^{2}\right) \rightarrow S^{4}=\mathbb{P}_{\mathbb{H}}\left(\mathbb{H}^{2}\right)$ be the "Hopf mapping" which assigns to a complex line the quaternion line containing it. Define

$$
f: S^{4} \rightarrow \mathcal{Z}_{\mathbb{H}}^{2}\left(\mathbb{P}_{\mathbb{C}}\left(\mathbb{H}^{2}\right)\right)
$$

by setting $f(\ell)=p^{-1}(\ell)=$ " $\ell$ ". Then $[f]=x \in \pi_{4} \mathcal{Z}_{\mathbb{H}}^{\mathrm{ev}} \cong \mathbb{Z}$ is the generator. Furthermore, under the ring homomorphism (38) one has

$$
\Phi_{*}(x)=s^{2}
$$

and so $\Phi_{*}\left(x^{m}\right)=s^{2 m}$ for all $m$. Similarly, let $\lambda$ denote the generator of $\pi_{0} \mathcal{Z}_{\mathbb{H}}^{\text {odd }} \cong \mathbb{Z}$. Then $\Phi_{*}\left(x^{m} \lambda\right)=2 s^{2 m}$ for all $m$. In particular, $x^{m}$ and $x^{m} \lambda$ are additive generators for all $m$.

Proof Under the composition

$$
\pi_{4} \mathcal{Z}_{\mathbb{H}}^{2}\left(\mathbb{P}_{\mathbb{C}}^{3}\right) \stackrel{\Phi}{\rightarrow} \pi_{4} \mathcal{Z}_{\mathbb{C}}^{2}\left(\mathbb{P}_{\mathbb{C}}^{3}\right) \stackrel{\cong}{\rightrightarrows} H_{6}\left(\mathbb{P}_{\mathbb{C}}^{3} ; \mathbb{Z}\right)=\mathbb{Z}
$$

the class of $[f]$ goes to class of its "trace", which is the union of the lines parameterized by $f$ (cf [15, Section 9]). This trace is exactly $\mathbb{P}_{\mathbb{C}}^{3}$ whose class is the generator of $H_{6}$. It follows that $\Phi_{*}\left(x^{m}\right)$ must be the generator $s^{2}$.

By Theorem 2.3(ii) we know that every cycle in $\mathcal{Z}_{\mathbb{H}}^{2 q+1}$ is deformable to an averaged cycle, the homomorphism deg: $\pi_{0} \mathcal{Z}_{\mathbb{H}}^{\text {odd }} \rightarrow \mathbb{Z}$ given by projective degree has

$$
\operatorname{Im}\{\operatorname{deg}\}=2 \mathbb{Z}
$$

Now fix $x_{0} \in \mathbb{P}_{\mathbb{C}}(\mathbb{H})$ and consider the cycle $c_{0}=x_{0}+\mathbf{j} x_{0}$ whose component generates $\pi_{0} \mathcal{Z}_{\mathbb{H}}^{\text {odd }}$. Define $\tilde{f}: S^{4} \rightarrow \mathcal{Z}_{\mathbb{H}}^{3}\left(\mathbb{P}_{\mathbb{C}}^{5}\right)$ by $\widetilde{f}(t)=c_{0} \# f(t)$. Since $\tilde{f}$ is homotopic to twice the suspension of $f$ we see that $\tilde{f}$ has image $2 \mathbb{Z}$. The same holds for $\tilde{f} \wedge f \wedge \ldots \wedge f$. 
Proposition 7.2 Let 1 denote the generator of $\pi_{0} \mathcal{Z}_{\mathbb{H}}^{\text {ev }}=\mathbb{Z}$ and let $u$ and $v$ denote the generators of $\pi_{2} \mathcal{Z}_{\mathbb{H}}^{\text {ev }}=\mathbb{Z}_{2}$ and $\pi_{1} \mathcal{Z}_{\mathbb{H}}^{\text {odd }}=\mathbb{Z}_{2}$ respectively. Then 1 is the multiplicative unit and the following relations hold in the ring $\mathfrak{R}_{*}$ :

$$
\begin{aligned}
u \cdot \lambda & =0, & v \cdot \lambda=0, & u^{2}=0, \\
v^{2} & =0, & u \cdot v=0, & \lambda^{2}=4 .
\end{aligned}
$$

Proof That 1 is a multiplicative unit is an immediate consequence of the Quaternionic Suspension Theorem [14, Theorem 6.1]. From the paragraph above we see that $\lambda^{2}$ is represented by $c_{0} \# c_{0}$ which has degree 4 . Since $\operatorname{deg}: \pi_{0} \mathcal{Z}_{\mathbb{H}}^{\text {ev }} \rightarrow \mathbb{Z}$ is an isomorphism we conclude that $\lambda^{2}=4$. That $v^{2}=0$ is Lemma $\mathbf{7 . 3}$ below. All the remaining relations are trivial.

It remains to prove that $x^{m} u$ and $x^{m} v$ are additive generators for all $m>0$. To do this we will need explicit representatives for these classes.

Recall the isomorphisms

$$
\pi_{1} \mathcal{Z}_{\mathbb{H}}^{\text {odd }}=\pi_{1} \mathcal{Z}_{\mathbb{H}}^{1}\left(\mathbb{P}_{\mathbb{C}}(\mathbb{H})\right)=\pi_{1} \mathcal{Z}_{0}\left(\mathbb{P}_{\mathbb{C}} / \mathbb{Z}_{2}\right)=H_{1}\left(\mathbb{P}_{\mathbb{C}} / \mathbb{Z}_{2} ; \mathbb{Z}\right)=H_{1}\left(\mathbb{P}_{\mathbb{R}} ; \mathbb{Z}\right)=\mathbb{Z}_{2} .
$$

Unravelling these isomorphisms one sees that the generator $v$ of $\pi_{1} \mathcal{Z}_{\mathbb{H}}^{\text {odd }}$ is represented by the following map. Let $\ell:[0, \pi] \rightarrow S^{2}$ be the standard longitudinal curve joining the north and south poles. Under the identification $S^{2}=\mathbb{P}_{\mathbb{C}}^{1}$, we consider $\ell(t)$ to be a complex line in $\mathbb{C}^{2}=\mathbb{H}$. Note that for $\ell \in \mathbb{P}_{\mathbb{C}}^{1} \subset \mathcal{Z}_{0}\left(\mathbb{P}_{\mathbb{C}}^{1}\right)=\mathcal{Z}_{\mathbb{H}}^{1}\left(\mathbb{P}_{\mathbb{C}}(\mathbb{H})\right)$ we have that

$$
\mathbf{j}(\ell)=\ell^{\perp}=\text { the antipodal image of the point } \ell .
$$

We now define $\phi: S^{1} \rightarrow \pi_{1} \mathcal{Z}_{\mathbb{H}}^{1}\left(\mathbb{P}_{\mathbb{C}}(\mathbb{H})\right)$ by

$$
\phi(t)=\ell(t)+\ell^{\perp}(t)-\left(\ell_{0}+\ell_{0}^{\perp}\right) .
$$

where $\ell_{0}=\ell(0)$. By (40) we see that $\phi$ is a map into $\mathbf{j}$-averaged cycles and $\phi(0)=\phi(\pi)$.

Lemma 7.3 The map $\phi$ represents the generator $v$ of $\pi_{1} \mathcal{Z}_{\mathbb{H}}^{\text {odd }}$, and $v^{2}=0$ in $\pi_{2} \mathcal{Z}_{\mathbb{H}}^{\text {odd }}$.

Proof The first statement follows from the paragraph above. For the second note that

$$
\begin{aligned}
\phi(s) \# \phi(t)= & \left\{\ell(s)+\ell^{\perp}(s)-\left(\ell_{0}+\ell_{0}^{\perp}\right)\right\} \#\left\{\ell(t)+\ell^{\perp}(t)-\left(\ell_{0}+\ell_{0}^{\perp}\right)\right\} \\
= & \left\{\left(\ell(s)-\ell_{0}\right) \#\left(\ell(t)-\ell_{0}\right)+\left(\ell^{\perp}(s)-\ell_{0}^{\perp}\right) \#\left(\ell^{\perp}(t)-\ell_{0}^{\perp}\right)\right\}+ \\
& \quad\left\{\left(\ell(s)-\ell_{0}\right) \#\left(\ell^{\perp}(t)-\ell_{0}^{\perp}\right)+\left(\ell^{\perp}(s)-\ell_{0}^{\perp}\right) \#\left(\ell(t)-\ell_{0}\right)\right\}
\end{aligned}
$$




$$
\begin{aligned}
& =\left\{\left(\ell(s)-\ell_{0}\right) \#\left(\ell(t)-\ell_{0}\right)+\mathbf{j}\left(\ell(s)-\ell_{0}\right) \#\left(\ell(t)-\ell_{0}\right)\right\}+ \\
& \quad\left\{\left(\ell(s)-\ell_{0}\right) \#\left(\ell^{\perp}(t)-\ell_{0}^{\perp}\right)+\mathbf{j}\left(\ell(s)-\ell_{0}\right) \#\left(\ell^{\perp}(t)-\ell_{0}^{\perp}\right)\right\} \\
& \stackrel{\text { def }}{=} A(s, t)+B(s, t) .
\end{aligned}
$$

It is straightforward to see that $A$ and $B$ are homotopic as maps from $S^{2}$ into $\mathcal{Z}_{\mathbb{H}}^{2}\left(\mathbb{P}_{\mathbb{C}}\left(\mathbb{H}^{2}\right)\right)$. Hence, $v^{2}=2[A]=0$ in $\pi_{2} \mathcal{Z}_{\mathbb{H}}^{\text {ev }}=\mathbb{Z}_{2}$ as claimed.

\section{Proposition 7.4 Let}

$$
F=\phi \# f \# \ldots \# f: S^{1} \wedge S^{4} \wedge \cdots \wedge S^{4} \longrightarrow \mathcal{Z}_{\mathbb{H}}^{2 m+1}\left(\mathbb{P}_{\mathbb{C}}\left(\mathbb{H}^{2 m+1}\right)\right),
$$

where $f$ and $\phi$ are the maps from Proposition [7.1 and (411) respectively. Then $[F]=v \cdot x^{m}$ is the generator of $\pi_{4 m+1} \mathcal{Z}_{\mathbb{H}}^{\text {odd }}=\mathbb{Z}_{2}$.

Proof It suffices to show that $[F] \neq 0$. For this we will consider the graph of $F$ as in [15, 9.12ff]. To begin note that $F$ has the form

$$
\begin{aligned}
& F\left(t, \lambda_{1}, \ldots, \lambda_{m}\right) \\
& \qquad \begin{aligned}
=\left(\ell(t)+\ell^{\perp}(t)-\ell_{0}-\ell_{0}^{\perp}\right) \#\left(\lambda_{1}\right. & \left.-\lambda_{0}\right) \#\left(\lambda_{2}-\lambda_{0}\right) \# \ldots \#\left(\lambda_{m}-\lambda_{0}\right) \\
& =\widetilde{F}\left(t, \lambda_{1}, \ldots, \lambda_{m}\right)+\mathbf{j} \widetilde{F}\left(t, \lambda_{1}, \ldots, \lambda_{m}\right)
\end{aligned}
\end{aligned}
$$

where $\widetilde{F}=\left(\ell(t)-\ell_{0}\right) \#\left(\lambda_{1}-\lambda_{0}\right) \# \ldots \#\left(\lambda_{m}-\lambda_{0}\right)$ can be considered as a map into cycles on $X \stackrel{\text { def }}{=} \mathbb{P}_{\mathbb{C}}\left(\mathbb{H}^{2 m+1}\right) / \mathbb{Z}_{2}$. Now by Theorem 2.3 (ii) if $F$ is homotopic to 0 , then it is homotopic to zero through $\mathbf{j}$-averaged cycles, and therefore $\widetilde{F}$ is homotopic to 0 as a map into cycles on $X$.

To show $\widetilde{F}$ is not homotopic to 0 we consider its graph $\Gamma$ in $S^{4 m+1} \times X$ and show that its projection $\left[\operatorname{pr}_{*} \Gamma\right] \neq 0$ in $H_{8 m+1}\left(X ; \mathbb{Z}_{2}\right)$ (see [15, Lemma 9.12]). Now we see that

$$
\operatorname{pr}_{*} \Gamma=\bigcup_{t, \lambda_{1}, \ldots, \lambda_{m}} \ell(t) \# \lambda_{1} \# \ldots \# \lambda_{m}+\epsilon \equiv G+\epsilon
$$

where $\epsilon$ consists of terms which have dimension strictly less than $8 m+1$ and can be ignored.

Suppose now that $q=\left(q_{0}, q_{1}, \ldots, q_{m}\right) \in \mathbb{H} \oplus \mathbb{H}^{2} \oplus \cdots \oplus \mathbb{H}^{2}$ is any point such that $q_{j} \neq 0$ for all $j$. Then there is exactly one subspace from the family for $G$ which contains $q$. Such a point is clearly a regular point of the cycle $G$.

We now consider a great circular curve $\mu:[0, \pi] \rightarrow \mathbb{P}_{\mathbb{R}}^{1}$ which intersects the great circle defined by $\ell(t)$ above, transversely (in one point). We then set

$$
\gamma(s)=\left(\mu(s), q_{0}, \ldots, q_{0}\right) \quad \text { where } q_{0}=(1,0) \in \mathbb{H}^{2} .
$$


This closed curve intersects $G$ in exactly one point. That point is a regular point of $G$ and the intersection is transversal. Thus by [15. Lemma 9.13] the cycle $G$ is not homologous to zero and the proposition is proved.

To complete our analysis of the ring structure we need an explicit representative of the generator $u$ of $\pi_{2} \mathcal{Z}_{\mathbb{H}}^{\text {ev }}$. Define the map

$$
\psi: S^{2}=\mathbb{P}_{\mathbb{C}}^{1}(\mathbb{H}) \longrightarrow \mathcal{Z}_{\mathbb{H}}^{2}\left(\mathbb{P}_{\mathbb{C}}\left(\mathbb{H}^{2}\right)\right)
$$

by $\psi=\widetilde{\psi}+\mathbf{j} \widetilde{\psi}$ where

$$
\widetilde{\psi}(\ell)=\ell_{0} \oplus \ell-\ell_{0} \oplus \ell_{0} \quad \text { in } \mathbb{H} \oplus \mathbb{H} .
$$

To understand this map we shall examine a basis for the homology of $Q\left(\mathbb{H}^{n}\right) / \mathbb{Z}_{2}$. Recall the following notation from Section [6.

$$
X^{2 n-1}=\mathbb{P}_{\mathbb{C}}\left(\mathbb{H}^{n}\right) / \mathbb{Z}_{2} \quad \text { and } \quad Y^{2 n}=Q\left(\mathbb{H}^{n}\right) / \mathbb{Z}_{2}
$$

For $k \leq n$ there are embeddings

$$
X^{2 k-1} \subset Y^{2 n} \quad \text { and } \quad Y^{2 k} \subset Y^{2 n}
$$

where the second comes from the linear inclusion $\mathbb{H}^{k} \subset \mathbb{H}^{n}$ and $X^{2 k-1} \subset Y^{2 k}$ comes from the 0 -section of $\mathcal{O}(2)$. The analytic subsets $Y^{2 k}$ have oriented regular sets and define integral cycles which generate $H_{4 k}\left(Y^{2 n} ; \mathbb{Z}\right)=\mathbb{Z}$. Each $X^{2 k-1}$ is a smooth nonorientable submanifold of the regular set of $Y^{2 n}$. For each $k$ consider the subspace $U^{2 k+1}=\mathbb{C} \oplus \mathbb{H}^{k} \subset \mathbb{H}^{n-k} \oplus \mathbb{H}^{k}$, let $Q\left(U^{2 k+1}\right) \subset Q\left(\mathbb{H}^{n}\right)$ be the Thom space of $\mathcal{O}_{\mathbb{P}\left(U^{2 k+1}\right)}(2)$, and set

$$
Z^{2 k+1}=\pi\left(Q\left(U^{2 k+1}\right)\right)
$$

where $\pi: Q\left(\mathbb{H}^{n}\right) \rightarrow Y^{2 n}$ is the projection. Each $Z^{2 k+1}$ is an oriented analytic subvariety which defines an integral cycle in $Y^{2 n}$.

Lemma 7.5 For each $k<n$ the class $\left[Z^{2 k+1}\right]$ in $H_{4 k+2}\left(Y^{2 n} ; \mathbb{Z}\right)=\mathbb{Z}_{2}$ is nonzero.

Proof Note that $X^{2(n-k)-1}$ intersects $Z^{2 k+1}$ transversely in exactly one point (in its regular set). The Lemma now follows from [15, Lemma 9.13].

Proposition 7.6 Fix $m \geq 0$ and set

$$
F=\psi \# f \# \ldots \# f: S^{2} \wedge S^{4} \wedge \cdots \wedge S^{4} \longrightarrow \mathcal{Z}_{\mathbb{H}}^{2 m+2}\left(\mathbb{P}_{\mathbb{C}}\left(\mathbb{H}^{2 m+2}\right)\right),
$$

where $f$ and $\psi$ are the maps from Proposition 7.1 and (42) respectively. Then $[F]=u \cdot x^{m}$ is the generator of $\pi_{4 m+2} \mathcal{Z}_{\mathbb{H}}^{\mathrm{ev}}=\mathbb{Z}_{2}$. 
Proof Applying the homotopy equivalence $\not{Z}=\not_{\mathcal{O}(2)}$ of Proposition 6.1 gives a map

$$
\widehat{F}=\not 4 \circ F: S^{4 m+2} \longrightarrow \mathcal{Z}^{2 m+2}\left(Q\left(\mathbb{H}^{2 m+2}\right)\right)^{\mathrm{av}}
$$

which splits as

$$
\widehat{F}=\widetilde{F}+\widetilde{\mathbf{j}} \widetilde{F}
$$

where $\tilde{\mathbf{j}}$ is the real structure on $Q\left(\mathbb{H}^{2 m+2}\right)$. Proceeding in strict analogy with the proof of Proposition 7.4 we are reduced to showing that the cycle

$$
\begin{aligned}
G & =\pi\left\{\bigcup_{\ell, \lambda_{1}, \ldots, \lambda_{m}} \not\left(\ell_{0} \# \ell \# \lambda_{1} \# \ldots \# \lambda_{m}\right)\right\} \\
& =\pi\left\{\not{\not}\left(U^{2 m+3}\right)\right\}=\pi\left(Q\left(U^{2 m+3}\right)\right)=Z^{2 m+3}
\end{aligned}
$$

is not 0 in $H_{4 m+6}\left(Y^{2 m+2} ; \mathbb{Z}_{2}\right)$. This was proved in Lemma 7.5 .

This completes the proof of Theorem 3.4

\section{Quaternionic projective varieties}

The main theme of this paper is the study of spaces of quaternionic cycles. Their structure turns out to be surprising and rich. However the geometry of quaternionic varieties themselves is of independent interest. In this and subsequent sections we will examine these varieties and show how our cycle spaces provide invariants for their study.

Definition 8.1 A quaternionic projective variety is an algebraic subvariety $X \subset \mathbb{P}_{\mathbb{C}}\left(\mathbb{H}^{n}\right)$ which is invariant under the quaternionic involution $\mathbf{j}$. A quaternionic morphism of quaternionic projective varieties is a morphism which commutes with $\mathbf{j}$.

As we have seen, there are many quaternionic varieties. The abelian group they generate has the rich homotopy structure determined above. It is useful to look at some specific examples.

Example 8.2 Fermat varieties Choose coordinates $\left(q_{1}, \ldots, q_{n}\right)$ for $\mathbb{H}^{n}$ and write

$$
q_{k}=z_{k}+w_{k} \cdot j \quad \text { where } z_{k}, w_{k} \in \mathbb{C}
$$


for all $k$. Then the Fermat variety

$$
F(2 m)=\left\{(z, w) \in \mathbb{C}^{n} \oplus \mathbb{C}^{n} \cdot j=\mathbb{H}^{n}: \sum_{k} z_{k}^{2 m}+w_{k}^{2 m}=0\right\}
$$

is a quaternionic variety for all $m \geq 1$. This includes the K3-surface $F(4) \subset$ $\mathbb{P}_{\mathbb{C}}\left(\mathbb{H}^{2}\right)$.

Example 8.3 Quaternionic divisors Let $\operatorname{Div}_{2 m} \cong \mathbb{P}_{\mathbb{C}}^{\left({ }^{2(n+m)}\right)-1}$ be the space of divisors of degree $2 m$ on $\mathbb{P}_{\mathbb{C}}\left(\mathbb{H}^{n}\right)$. Then $\mathbf{j}$ induces a linear antiholomorphic involution on $\operatorname{Div}_{2 m}$ whose fixed-point set is nonempty by Example 8.2. Thus the subset $\operatorname{Div}_{2 m}^{\mathbb{H}} \subset \operatorname{Div}_{2 m}$ of quaternionic divisors is a real form

$$
\left.\operatorname{Div} \underset{2 m}{\mathbb{H}} \cong \mathbb{P}_{\mathbb{R}}^{(2(n+m)}{ }^{2(n+m)} \subset 1 \subset \mathbb{P}_{\mathbb{C}}^{(2(n+m)}{ }^{2 n}\right)-1
$$

In general $\mathbf{j}$ induces a real structure on $\operatorname{Div}_{\text {even }}$ and a quaternionic structure on Div $_{\text {odd }}$.

The evenness of degree here is required by Theorem 2.3(ii). Here is an elementary proof.

Proposition 8.4 Let $X \subset \mathbb{P}_{\mathbb{C}}\left(\mathbb{H}^{n}\right)$ be a quaternionic projective variety of odd (complex) codimension. Then the degree of $X$ is even.

Proof Let $\operatorname{codim}(X)=2 q-1$ and choose a quaternionic linear subspace $V \subset \mathbb{H}^{n}$ with $\operatorname{dim}_{\mathbb{H}}=q$ such that $\mathbb{P}(V)$ meets $X$ transversely at regular points. The existence of such a $V$ follows from the transitivity of $S p_{n}$ on $\mathbb{P}_{\mathbb{C}}\left(\mathbb{H}^{n}\right)$ and Sard's Theorem for families (cf [1], Appendix A]). Now $\mathbb{P}(V) \cap X$ is $\mathbf{j}$-invariant, and so $\operatorname{deg}(X)=\#(\mathbb{P}(V) \cap X)$ is even.

Example 8.5 Quaternionic rational normal curves Notice that the mapping

$$
\mathbb{P}_{\mathbb{C}}(\mathbb{H}) \longrightarrow \mathbb{P}_{\mathbb{C}}\left(\mathbb{H}^{n}\right)
$$

given by

$$
(z, w) \mapsto(Z, W)=\left(z^{2 n-1}, z^{2 n-2} w, \ldots, z^{n} w^{n-1} ; w^{2 n-1}, w^{2 n-2} z, \ldots, w^{n} z^{n-1}\right)
$$

is a quaternionic morphism. Its image is a $\mathbf{j}$-invariant rational normal curve. The moduli space of such curves is a real form for the space of all rational normal curves in $\mathbb{P}_{\mathbb{C}}\left(\mathbb{H}^{n}\right)$. 
Example 8.6 Quaternionic Veronese and Segré embeddings More generally one can check that there is a nonempty subspace of quaternionic Veronese embeddings

$$
\mathbb{P}_{\mathbb{C}}\left(\mathbb{H}^{n}\right) \longrightarrow \mathbb{P}_{\mathbb{C}}\left(\operatorname{Sym}_{\mathbb{C}}^{2 k+1} \mathbb{H}^{n}\right)
$$

in any odd degree $2 k+1$. This is also true of the Segré embeddings

$$
\mathbb{P}_{\mathbb{C}}\left(\mathbb{H}^{n_{1}}\right) \times \cdots \times \mathbb{P}_{\mathbb{C}}\left(\mathbb{H}^{n_{k}}\right) \longrightarrow \mathbb{P}_{\mathbb{C}}\left(\mathbb{H}^{n_{1}} \otimes_{\mathbb{C}} \cdots \otimes_{\mathbb{C}} \mathbb{H}^{n_{k}}\right)
$$

for all $k$ odd.

Example 8.7 General quaternionic curves As we saw in Section 2 quaternionic curves can be thought of as "nonorientable" algebraic curves over $\mathbb{C}$.

Theorem 8.8 (Intrinsic characterization of quaternionic projective manifolds) Let $X$ be a compact Kähler manifold with an antiholomorphic involution $j: X \rightarrow X$. Suppose there exists a positive holomorphic line bundle $\pi: L \rightarrow X$ which admits an anti-linear bundle map $\widetilde{j}: E \rightarrow E$ such that

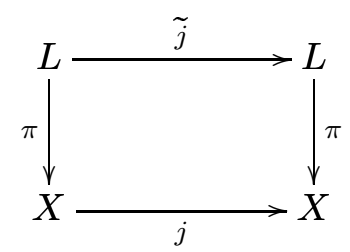

commutes, and $\widetilde{j}^{2}=-\mathrm{Id}$. Then there exists a $j$-equivariant holomorphic embedding $\Phi: X \hookrightarrow \mathbb{P}_{\mathbb{C}}\left(\mathbb{H}^{N}\right)$ for some $N$.

Proof Let $W_{k}$ denote the space of all holomorphic cross-sections of the bundle $L^{\otimes k}$. By the fundamental theorem of Kodaira for all $k$ sufficiently large, the mapping

$$
\Phi: X \longrightarrow \mathbb{P}\left(W_{k}\right)^{*} \quad \text { given by } \quad \Phi(x)=\operatorname{ker}\{\sigma \mapsto \sigma(x)\}
$$

is a well-defined projective embedding. For $k$ odd the bundle $L^{\otimes k}$ is quaternionic and there is a quaternionic structure $\mathbf{j}$ on $W_{k}$ defined by setting $\mathbf{j}(\sigma) \equiv$ $\widetilde{j}^{-1} \circ \sigma \circ j$. Note that $\Phi(j x)=\operatorname{ker}\{\sigma \mapsto \sigma(j x)\}=\operatorname{ker}\left\{\sigma \mapsto \widetilde{j}^{-1} \circ \sigma \circ j(x)\right\}=$ $\{\mathbf{j}(\sigma): \sigma(x)=0\}=\mathbf{j}(\Phi(x))$, which proves the $j$-equivariance.

This theorem motivates the definition of a quaternionic topological space given below. 


\section{Quaternionic algebraic cocycles and morphic co- homology}

We now want to consider families of quaternionic varieties $\pi: \mathcal{F} \rightarrow X$ over a parameter space $X$. Such families generalize the concept of a quaternionic vector bundle. We will begin in the algebraic category and adopt the viewpoint of algebraic cocyles developed in [9]. Much of that theory carries over to the quaternionic case.

Recall the Chow monoid

$$
\mathcal{C}^{q}\left(\mathbb{P}_{\mathbb{C}}\left(\mathbb{H}^{n}\right)\right)=\coprod_{d \geq 0} \mathcal{C}_{d}^{q}\left(\mathbb{P}_{\mathbb{C}}\left(\mathbb{H}^{n}\right)\right)
$$

where $\mathcal{C}_{d}^{q}\left(\mathbb{P}_{\mathbb{C}}\left(\mathbb{H}^{n}\right)\right)$ is the Chow variety of effective algebraic cycles of degree $d$ and codimension $q$ in $\mathbb{P}_{\mathbb{C}}\left(\mathbb{H}^{n}\right)$. The map $\mathbf{j}$ induces an involution, also denoted $\mathbf{j}$, on each of these varieties. Let $\mathcal{C}_{\mathbb{H}}^{q}\left(\mathbb{P}_{\mathbb{C}}\left(\mathbb{H}^{n}\right)\right) \subset \mathcal{C}^{q}\left(\mathbb{P}_{\mathbb{C}}\left(\mathbb{H}^{n}\right)\right)$ denote the submonoid of $\mathbf{j}$-fixed cycles.

Definition 9.1 Let $X$ be a quaternionic variety (or more generally any real variety with involution given by the action of $\mathrm{Gal}(\mathbb{C} / \mathbb{R}))$. By a quaternionic algebraic cocycle on $X$ we mean a $\mathbf{j}$-equivariant morphism

$$
\varphi: X \longrightarrow \mathcal{C}^{q}\left(\mathbb{P}_{\mathbb{C}}\left(\mathbb{H}^{n}\right)\right)
$$

for some $n$. The set of all quaternionic algebraic cocycles forms an abelian monoid

$$
\operatorname{Mor}_{\mathbb{H}}\left(X ; \mathcal{C}^{q}\left(\mathbb{P}_{\mathbb{C}}\left(\mathbb{H}^{n}\right)\right)\right.
$$

whose group completion will be denoted by $\operatorname{Mor}_{\mathbb{H}}\left(X ; \mathcal{Z}^{q}\left(\mathbb{P}_{\mathbb{C}}\left(\mathbb{H}^{n}\right)\right)\right.$. Note that each cocycle $\varphi \in \operatorname{Mor}_{\mathbb{H}}\left(X ; \mathcal{C}^{q}\left(\mathbb{P}_{\mathbb{C}}\left(\mathbb{H}^{n}\right)\right)\right.$ gives rise to a mapping

$$
\widetilde{\varphi}: X / \mathbb{Z}_{2} \longrightarrow \mathcal{Z}_{\mathbb{H}}^{q}\left(\mathbb{P}_{\mathbb{C}}\left(\mathbb{H}^{n}\right)\right) \quad \text { where } \widetilde{\varphi}([x])=\varphi(x)+\mathbf{j} \varphi(x) .
$$

Example 9.2 The fundamental class Let $\varphi: X \subset \mathbb{P}_{\mathbb{C}}\left(\mathbb{H}^{n}\right)$ be a quaternionic variety. This inclusion is a quaternionic cocycle whose associated map

$$
\widetilde{\varphi}: X / \mathbb{Z}_{2} \rightarrow \mathcal{Z}_{\mathbb{H}}^{2 n-1}\left(\mathbb{P}_{\mathbb{C}}\left(\mathbb{H}^{n}\right)\right)
$$

is given by $\widetilde{\varphi}([x])=x+\mathbf{j} x$. With respect to the canonical splitting in Theorem 2.3(ii) this fundamental map can be viewed as

$$
\widetilde{\varphi}: X / \mathbb{Z}_{2} \longrightarrow \prod_{k=0}^{q} K(\mathbb{Z}, 4 k) \times \prod_{k=0}^{n} K\left(\mathbb{Z}_{2}, 4 k+1\right) .
$$


Define total classes

$$
\iota=1+\iota_{4}+\iota_{8}+\iota_{12}+\cdots \quad \text { and } \quad \widetilde{\iota}=\widetilde{\iota}_{2}+\widetilde{\iota}_{6}+\widetilde{\iota}_{10}+\widetilde{\iota}_{14}+\cdots
$$

where $\iota_{4 k} \in H^{4 k}(K(\mathbb{Z}, 4 k) ; \mathbb{Z})=\mathbb{Z}$ and $\widetilde{\iota}_{4 k+1} \in H^{4 k+1}\left(K\left(\mathbb{Z}_{2}, 4 k+1\right) ; \mathbb{Z}_{2}\right)=\mathbb{Z}_{2}$ denote the fundamental classes. Then associated to the embedding $\varphi: X \subset$ $\mathbb{P}_{\mathbb{C}}\left(\mathbb{H}^{n}\right)$ we have the classes

$$
\widetilde{\varphi}^{*} \iota \in H^{4 *}\left(X / \mathbb{Z}_{2} ; \mathbb{Z}\right) \quad \text { and } \quad \tilde{\varphi}^{*} \tau \in H^{4 *+1}\left(X / \mathbb{Z}_{2} ; \mathbb{Z}_{2}\right) .
$$

When $X=\mathbb{P}_{\mathbb{C}}\left(\mathbb{H}^{n}\right)$, these classes are nonzero in every dimension.

For a general quaternionic variety $X \subset \mathbb{P}_{\mathbb{C}}\left(\mathbb{H}^{n}\right)$ of dimension $2 q-1$ we can find quaternionic projection $\mathbb{P}_{\mathbb{C}}\left(\mathbb{H}^{n}\right)-\mathbb{P}_{\mathbb{C}}\left(\mathbb{H}^{n-q-1}\right) \longrightarrow \mathbb{P}_{\mathbb{C}}\left(\mathbb{H}^{q}\right)$ which restricts to give a quaternionic morphism $X \longrightarrow \mathbb{P}_{\mathbb{C}}\left(\mathbb{H}^{q}\right)$. The classes 45 for $X$ are the pull-backs of those for $\mathbb{P}_{\mathbb{C}}\left(\mathbb{H}^{q}\right)$.

Example 9.3 The quaternionic Gauss map Let $X \subset \mathbb{P}_{\mathbb{C}}\left(\mathbb{H}^{n}\right)$ be a smooth quaternionic variety of codimension $-q$, and consider the quaternionic morphism

$$
\gamma: X \longrightarrow G_{\mathbb{C}}^{q}\left(\mathbb{P}_{\mathbb{C}}\left(\mathbb{H}^{n}\right)\right) \quad \text { where } \gamma(x)=\left[T_{x} X\right] .
$$

The associated characteristic map $\widetilde{\gamma}: X / \mathbb{Z}_{2} \longrightarrow \mathcal{Z}_{\mathbb{H}}^{q}\left(\mathbb{P}_{\mathbb{C}}\left(\mathbb{H}^{n}\right)\right)$ can be rewritten in terms of the canonical splitting in Theorem 2.3 as a mapping

$$
\tilde{\gamma}: X / \mathbb{Z}_{2} \longrightarrow \begin{cases}\prod_{j=0}^{r} K(\mathbb{Z}, 4 j) \times \prod_{j=0}^{r-1} K\left(\mathbb{Z}_{2}, 4 j+2\right) & \text { if } q=2 r, \\ \prod_{j=0}^{r} K(\mathbb{Z}, 4 j) \times \prod_{j=0}^{r} K\left(\mathbb{Z}_{2}, 4 j+1\right) & \text { if } q=2 r+1 .\end{cases}
$$

Let $\iota$ and $\tau$ be defined as in Example 9.2 when $q=2 r$ and let them be the obvious analogues when $q=2 r+1$. Then we can define the normal quaternionic characteristic classes of $X$ :

$$
\widetilde{\gamma}^{*}(\iota) \in H^{4 *}\left(X / \mathbb{Z}_{2} ; \mathbb{Z}\right) \text { and } \widetilde{\gamma}^{*}(\widetilde{\iota}) \in \begin{cases}H^{4 *+2}\left(X / \mathbb{Z}_{2} ; \mathbb{Z}_{2}\right) & \text { if } q=2 r \\ H^{4 *+1}\left(X / \mathbb{Z}_{2} ; \mathbb{Z}_{2}\right) & \text { if } q=2 r+1\end{cases}
$$

As an example consider the Fermat variety $F(2) \subset \mathbb{P}_{\mathbb{C}}\left(\mathbb{H}^{n}\right)$. Its Gauss map

$$
\gamma: F(2) \longrightarrow \mathbb{P}_{\mathbb{C}}^{*}\left(\mathbb{H}^{n}\right)=G_{\mathbb{C}}^{1}\left(\mathbb{P}_{\mathbb{C}}\left(\mathbb{H}^{n}\right)\right)
$$

is essentially the identity $(F(2)$ is self-dual). The associated map

$$
\widetilde{\gamma}: F(2) / \mathbb{Z}_{2} \longrightarrow \mathcal{Z}_{\mathbb{H}}^{1}\left(\mathbb{P}_{\mathbb{C}}\left(\mathbb{H}^{n}\right)\right) \cong K\left(\mathbb{Z}_{2}, 1\right)
$$

is easily seen to be nontrivial on $\pi_{1}$ and so $\widetilde{\gamma}^{*}(\widetilde{\iota}) \neq 0$.

Example 9.4 Let $X \subset \mathbb{P}_{\mathbb{C}}\left(\mathbb{H}^{n}\right)$ be an irreducible hypersurface of degree $d>1$. The we can define

$$
\varphi: X \longrightarrow \mathcal{C}_{d^{2}}^{2}\left(\mathbb{P}_{\mathbb{C}}\left(\mathbb{H}^{n}\right)\right)
$$

by $\varphi(x)=X \bullet T_{x} X$ where "•" is the intersection product ([10]). 
Example 9.5 Consider the product quaternionic variety

$$
X=x_{0} \times \mathbb{P}_{\mathbb{C}}(\mathbb{H}) \amalg\left(\mathbf{j} x_{0}\right) \times \mathbb{P}_{\mathbb{C}}(\mathbb{H}) \cong \mathbb{Z}_{2} \times S^{2}
$$

where $x_{0}$ is a point, and the map $f: X \rightarrow G_{\mathbb{C}}^{2}\left(\mathbb{P}_{\mathbb{C}}\left(\mathbb{H}^{2}\right)\right)$ given by

$$
f\left(x_{0} \times \ell\right)=\ell_{0} \oplus \ell \quad \text { and } \quad f\left(\mathbf{j} x_{0} \times \ell\right)=\mathbf{j} \ell_{0} \oplus \ell \quad \text { in } \mathbb{H} \oplus \mathbb{H}
$$

where $\ell_{0} \subset \mathbb{H} \oplus\{0\} \subset \mathbb{H}^{2}$ is a fixed complex line. Then the map

$$
\tilde{f}: X / \mathbb{Z}_{2}=S^{2} \longrightarrow \mathcal{Z}_{\mathbb{H}}^{2}
$$

represents the generator of $\pi_{2} \mathcal{Z}_{\mathbb{H}}^{2}=\mathbb{Z}_{2}$ as we saw in Proposition [7.6.

Example 9.6 Suppose $X \subset \mathbb{P}_{\mathbb{C}}\left(\mathbb{H}^{2}\right)$ is a quaternionic algebraic surface of degree $2 k$ which contains no quaternionic lines. Then there is a well-defined continuous map

$$
\psi_{X}: S^{4} \longrightarrow \mathcal{Z}_{\mathbb{H}}^{3}
$$

given by $\psi_{X}(p)=\pi^{-1}(p) \bullet X$ where $\pi: \mathbb{P}_{\mathbb{C}}\left(\mathbb{H}^{2}\right) \longrightarrow S^{4}$ is the Hopf fibration (See Proposition [7.1) and "•" denotes intersection product (cf [10). Now recall the isomorphism $\tau: \mathcal{Z}_{\mathbb{H}}^{3}\left(\mathbb{P}_{\mathbb{C}}\left(\mathbb{H}^{2}\right)\right) \longrightarrow \mathcal{Z}_{0}\left(\mathbb{P}_{\mathbb{C}}\left(\mathbb{H}^{2}\right) / \mathbb{Z}_{2}\right)$ and set $\psi_{X}=\tau \circ \psi_{X}$. Then if $\tilde{\pi}: \mathcal{Z}_{0}\left(\mathbb{P}_{\mathbb{C}}\left(\mathbb{H}^{2}\right) / \mathbb{Z}_{2}\right) \longrightarrow \mathcal{Z}_{0}\left(S^{4}\right)$ is the extension of the map $\mathbb{P}_{\mathbb{C}}\left(\mathbb{H}^{2}\right) / \mathbb{Z}_{2} \longrightarrow S^{4}$ then $\widetilde{\pi} \circ \widetilde{\psi}_{X}=k \cdot$ Id. Therefore

$$
[\psi]=k \in \pi_{4} \mathcal{Z}_{\mathbb{H}}^{3} .
$$

\section{Linear cocycles}

The cocycles introduced in Definition 9.1 are particularly interesting when $\varphi(x)$ is a linear subspace for all $x$.

Definition 10.1 Let $X$ be as in Definition 9.1 By an effective quaternionic bundle of dimension $q$ on $X$ we mean a $\mathbf{j}$-equivariant morphism $f: X \rightarrow$ $G_{\mathbb{C}}^{q}\left(\mathbb{P}_{\mathbb{C}}\left(\mathbb{H}^{N}\right)\right)$ for some $N$.

Such a morphism corresponds to an algebraic vector bundle $E \rightarrow X$ which is generated by its global sections and which is equipped with an anti-linear bundle map $\widetilde{j}: E \rightarrow E$ which covers $j: X \rightarrow X$ and satisfies $\widetilde{j}^{2}=-$ Id. These linear cocycles form a submonoid under the algebraic join operation $(\cong$ direct sum in this case). The homotopy groups of its group completion are interesting invariants of the variety. In fact this group completion can be expanded to a generalized equivariant cohomology theory attached to $X$. (See [6].) 
Note that to any quaternionic bundle $f: X \rightarrow G_{\mathbb{C}}^{q}\left(\mathbb{P}_{\mathbb{C}}\left(\mathbb{H}^{N}\right)\right)$ there is an associated mapping

$$
\tilde{f}: X / \mathbb{Z}_{2} \longrightarrow \mathcal{Z}_{\mathbb{H}}^{q}\left(\mathbb{P}_{\mathbb{C}}\left(\mathbb{H}^{n}\right)\right)
$$

and we get classes $\tilde{f}^{*}(\iota)$ and $\tilde{f}^{*}(\widetilde{\iota})$ as in (46) above. For the full theory of characteristic classes in this setting one must consider the full equivariant theory. This is done in detail in [5]

\section{Quaternionic spaces, quaternionic bundles and $K H$-theory}

The notions of quaternionic vector bundles varieties can be generalized to the topological category. Recall that a space with a real structure is a pair $(X, j)$ where $X$ is a topological space and $j: X \rightarrow X$ a continuous involution. The following notion was introduced by Johann Dupont [7].

Definition 11.1 A quaternionic vector bundle over a real space $(X, j)$ is a complex vector bundle $E \longrightarrow X$ together with an $\mathbb{C}$-anti-linear bundle map $\widetilde{j}: E \rightarrow E$ covering $j$ with $\widetilde{j}^{2}=-1$.

Such a pair $(E, \widetilde{j})$ with $\widetilde{j}^{2}=1$ is called a real bundle (cf [1]). Real bundles are classified by $\mathbb{Z}_{2}$-equivariant maps into the stabilized Grassmannian with its standard real structure [15]. The corresponding statement holds for quaternionic bundles.

Theorem 11.2 Let $X$ be a compact Hausdorff space with involution $j: X \rightarrow$ $X$. Then the isomorphism classes of quaternionic vector bundles of complex rank $q$ on $X$ are in one-to-one correspondence with $\mathbb{Z}_{2}$-homotopy classes of $\mathbb{Z}_{2}-\operatorname{maps} X \rightarrow G_{\mathbb{C}}^{q}\left(\mathbb{P}_{\mathbb{C}}\left(\mathbb{H}^{\infty}\right)\right)$.

Proof This is a direct adaptation of the standard arguments (cf [21]).

Corollary 11.3 Let $E \rightarrow X$ be a quaternionic vector bundle classified by a $j$-equivariant map $f: X \rightarrow G_{\mathbb{C}}^{q}\left(\mathbb{P}_{\mathbb{C}}\left(\mathbb{H}^{\infty}\right)\right)$. Then the classes $\tilde{f}^{*}(\iota)$ and $\widetilde{f}^{*}(\widetilde{\iota})$ defined as in (46) above, depend only on the isomorphism class of $E$.

The Grothendieck group $K R(X)$ of real bundles on $X$ are the basis of Atiyah's real $K$-theory [1. The Grothendieck group $K H(X)$ of quaternionic bundles on $X$ form an analogous quaternionic $K$-theory [7]. However, this theory 
is not multiplicative. The tensor product of two quaternionic bundles is not quaternionic ; it is real. However, the combined theory $K R(X) \oplus K H(X)$ has a product structure, and interestingly there is an isomorphism

$$
K R\left(X \times \mathbb{P}_{\mathbb{C}}(\mathbb{H})\right) \cong K R(X) \oplus K H(X)
$$

observed by Dupont [7]. In a subsequent paper [8] Dupont asks whether there is an appropriate theory of characteristic classes for quaternionic bundles and KH-theory. An answer to the analogous question for real bundles and KRtheory was given by dos Santos [4. The answer in the quaternionic case has been recently given by dos Santos and Lima-Filho [5] who continued this study of the space of quaternionic algebraic cycles.

Definition 11.4 A quaternionic space is a triple $(X, j, \mathcal{L})$ where $X$ is a topological space, $j: X \rightarrow X$ is an involution, and $\mathcal{L} \rightarrow X$ is a complex line bundle with quaternionic structure, that is, with a lifting of $j$ to an anti-linear bundle map $\tilde{j}: \mathcal{L} \longrightarrow \mathcal{L}$ such that $\widetilde{j}^{2}=-1$. Note that $j$ must be a free action.

Note 11.5 On a quaternionic projective variety $X$ we take $\mathcal{L}=\mathcal{O}(1)$.

Example 11.6 Quaternionifications of a space Any real space $(X, j)$, with possibly trivial involution, gives rise to a quaternionic space $X_{\mathbb{H}}$ as follows. Set $X_{\mathbb{H}}=\mathbb{Z}_{2} \times X$. Define $\mathbf{j}: X_{\mathbb{H}} \rightarrow X_{\mathbb{H}}$ by $\mathbf{j}(0, x)=(1, j x)$ and $\mathbf{j}(1, x)=(0, j x)$. Set $\mathcal{L}=X_{\mathbb{H}} \times \mathbb{C}$ and define $\tilde{\mathbf{j}}: \mathcal{L} \rightarrow \mathcal{L}$ by

$$
\widetilde{\mathbf{j}}(0, x, z)=(1, j x, \bar{z}) \quad \text { and } \quad \widetilde{\mathbf{j}}(1, x, z)=(0, j x,-\bar{z}) .
$$

This is the trivial quaternionification of $X$. There is a natural bijection between complex bundles on $X$ and quaternionic bundles on $X_{\mathbb{H}}$, and also between complex bundles on $X$ and real bundles on $X_{\mathbb{H}}$. In particular we have $K(X) \cong K R\left(X_{\mathbb{H}}\right) \cong K H\left(X_{\mathbb{H}}\right)$. In light of (47) above a more interesting quaternionification of $X$ is given by $X \times \mathbb{P}_{\mathbb{C}}(\mathbb{H})$ with $\mathcal{L}=\operatorname{pr}_{2}^{*} \mathcal{O}(1)$.

\section{The equivariant homotopy type of $\mathcal{Z}^{q}\left(\mathbb{P}_{\mathbb{C}}\left(\mathbb{H}^{\infty}\right)\right)$}

There are two distinct real structures on projective space (this reflects the fact that the Brauer group of $\mathbb{R}$ is $\mathbb{Z}_{2}$ ), and they in turn induce real structures on the groups of algebraic cycles. The first real structure, given by complex conjugation of homogeneous coordinates, was studied in part one of this work [15. The second, given by the quaternion involution on $\mathbb{P}_{\mathbb{C}}\left(\mathbb{H}^{n}\right)$ (called the Brauer-Severi variety), is studied here. It is natural to ask: what is the full 
equivariant homotopy type of the groups of algebraic cycles under the induced involutions?

Recall from [13] that the nonequivariant homotopy type of the group of cycles of codimension $q$ on $\mathbb{P}_{\mathbb{C}}^{n}$ is a product of Eilenberg-MacLane spaces $K(\mathbb{Z}, 0) \times$ $K(\mathbb{Z}, 2) \times \cdots \times K(\mathbb{Z}, 2 q)$. In his thesis 4 . Pedro dos Santos proved a beautiful, analogous result for cycles under the first involution. He showed that there is a $\mathbb{Z}_{2}$-equivariant homotopy equivalence

$$
\mathcal{Z}^{q}\left(\mathbb{P}_{\mathbb{C}}\left(\mathbb{C}^{n}\right)\right) \cong \prod_{k=0}^{q} K\left(\underline{\mathbb{Z}}, \mathbb{R}^{k, k}\right)
$$

for any $n>q$, where $K\left(\underline{\mathbb{Z}}, \mathbb{R}^{k, k}\right)$ denotes the Eilenberg-MacLane space classifying $\mathbb{Z}_{2}$-equivariant cohomology indexed at the representation $\mathbb{R}^{k, k}(=\mathbb{C}$ with complex conjugation) with coefficients in the constant Mackey functor $\underline{\mathbb{Z}}$.

Very recently dos Santos and Lima-Filho [5] established the corresponding result in the Brauer-Severi case. They prove that there are $\mathbb{Z}_{2}$-equivariant homotopy equivalences

$$
\begin{gathered}
\mathcal{Z}^{2 q-1}\left(\mathbb{P}_{\mathbb{C}}\left(\mathbb{H}^{n}\right)\right) \cong \prod_{k=0}^{q} \operatorname{Map}\left(\mathbb{P}_{\mathbb{C}}(\mathbb{H})_{+}, K\left(\underline{\mathbb{Z}}, \mathbb{R}^{2 k-1,2 k-1}\right)\right) \\
\mathcal{Z}^{2 q}\left(\mathbb{P}_{\mathbb{C}}\left(\mathbb{H}^{n}\right)\right) \cong \prod_{k=0}^{q} \operatorname{Map}\left(\mathbb{P}_{\mathbb{C}}(\mathbb{H})_{+}, K\left(\underline{\mathbb{Z}}, \mathbb{R}^{2 k, 2 k}\right)\right) .
\end{gathered}
$$

These spaces classify the $(\mathrm{k}, \mathrm{k})$-equivariant cohomology of $X \times \mathbb{P}_{\mathbb{C}}(\mathbb{H})$.

\section{References}

[1] M F Atiyah, K-theory and reality, Quart. J. Math. Oxford 17 (1966) 367-386 MathReview

[2] C P Boyer, H B Lawson Jr, P Lima-Filho, B Mann, M-L Michelson, Algebraic cycles and infinite loop spaces, Invent. Math. 113 (1993) 373-388 MathReview

[3] A Dold, R Thom, Quasifaserungen und unendliche symmetrische produkte, Ann. of Math. 67 (1956) 230-281 MathReview

[4] P F dos Santos, Algebraic cycles on real varieties and $\mathbb{Z} / 2$-equivariant homotopy theory, Proc. London Math. Soc. 86 (2003) 513-544 MathReview

[5] P F dos Santos, P Lima-Filho, Quaternionic algebraic cycles and reality, Trans. Amer. Math. Soc. 356 (2004) 4701-4736 MathReview 
[6] P F dos Santos, P Lima-Filho, Quaternionic $K$-theory for real varieties (in preparation)

[7] J Dupont, Symplectic bundles and KR-theory, Math. Scand. 24 (1969) 27-30 MathReview

[8] J Dupont, A note on characteristic classes for real vector bundles, preprint (1999)

[9] E Friedlander, H B Lawson Jr, A theory of algebraic cocycles, Ann. of Math. 136 (1992) 361-428 MathReview

[10] W Fulton, Intersection theory, Ergebnisse series (3) 2, Springer-Verlag, New York (1984) MathReview

[11] R Harvey, H B Lawson $\mathbf{J r}$, On boundaries of complex analytic varieties I, Ann. of Math. 102 (1975) 223-290 MathReview

[12] T-K Lam, Spaces of real algebraic cycles and homotopy theory, PhD thesis, SUNY, Stony Brook (1990)

[13] H B Lawson Jr, Algebraic cycles and homotopy theory, Ann. of Math. 129 (1989) 253-291 MathReview

[14] H B Lawson Jr, P Lima-Filho, M-L Michelsohn, On equivariant algebraic suspension, J. Algebraic Geom. 7 (1998) 627-650 MathReview

[15] H B Lawson Jr, P Lima-Filho, M-L Michelsohn, Algebraic cycles and the classical groups I: Real cycles, Topology 42 (2003) 467-506 MathReview

[16] H B Lawson Jr, M-L Michelsohn, Algebraic cycles, Bott periodicity, and the Chern characteristic map, from: "The Mathematical Heritage of Hermann Weyl (Durham, NC, 1987)", Proc. Sympos. Pure Math. 48, Amer. Math. Soc., Providence, RI, USA (1988) 241-264 MathReview

[17] P Lima-Filho, Lawson homology for quasiprojective varieties, Compositio Math. 84 (1992) 1-23 MathReview

[18] P Lima-Filho, Completions and fibrations for topological monoids, Trans. Amer. Math. Soc. 340 (1993) 127-146 MathReview

[19] P Lima-Filho, On the equivariant homotopy of free abelian groups on $G$-spaces and G-spectra, Math. Z. 224 (1997) 567-601 |MathReview

[20] J P May, $E_{\infty}$ ring spaces and $E_{\infty}$ ring spectra, Lecture Notes in Mathematics 577, Springer-Verlag, New York, USA (1977) MathReview

[21] J Milnor, J Stasheff, Characteristic Classes, Annals of Math. Studies 76, Princeton Univ. Press, Princeton, NJ, USA (1974) MathReview

[22] J Mostovoy, Quaternion flavored cycle spaces, ICMS preprint, Edinburgh University (1975) 